\title{
CROSS-RESISTANCE OF AZOLES, ECHINOCANDINS, FLUCYTOSINE AND AMPHOTERICIN B IN CLINICALLY IMPORTANT HYPHOMYCETES
}

\author{
SCHMALRECK A.F. ${ }^{1}$, FEGELER W. ${ }^{2}$, BECKER K. ${ }^{2}$, LASS-FLÖRL C. ${ }^{3}$ AND CZAIKA V. ${ }^{4}$ \\ ${ }^{1} \mathrm{MBS}$, Microbiology, 80086 Munich, Germany. \\ 2Institute of Medical Microbiology, University Hospital, 48149 Münster, Germany. \\ 3Division of Hygiene and Medical Microbiology, Medical University Innsbruck, 6020 Innsbruck, Austria. \\ ${ }^{4}$ Clinic for Dermatology, Venereology and Allergology, Charité, Campus Benjamin Franklin, 12203 Berlin, Germany. \\ *Corresponding Author: Email- muenchen-mbs@t-online.de
}

Received: April 28, 2014; Accepted: May 27, 2014

\begin{abstract}
The existing standardized micro-broth dilution methods for in vitro testing of moulds (CLSI, EUCAST) are referred to as reference methods and therefore not intended for routine testing. They are time-consuming and dependent on sporulating hyphomycetes. In this study a new, time saving and easy-to-perform method for inoculum preparation for routine susceptibility testing has been applied. It is independent of spore production and proofed to produce comparable results to the conidia based methods, indicating that it can be used for all types of hyphae-and/or conidia-forming fungi.

The MICs of amphotericin B, flucytosine, fluconazole, posaconazole, voriconazole, anidulafungin, caspofungin and micafungin of 198 moulds were determined with two different culture media (YST and RPMI 1640) according to the DIN microdilution assay, and compared to appropriate international studies. The "new" inocula with YST (DIN) and RPMI 1640 (EUCAST) medium showed similar MIC distributions for all moulds tested to the conidia method, with more than $92 \%$ of the MICs read at $24 \mathrm{~h}$ and $48 \mathrm{~h}$ within $\pm 1 \log _{2}$-dilution. Although azoles, flucytosine and amphotericin B showed comparable results, differences in echinocandin endpoints between different multicentre studies were determined. According to the literature, the minimum effective concentration (MEC) should be equivalent to the minimum inhibitory concentration (MIC). However, due to the encountered bias in echinocandin-endpoint determinations this has to be questioned. Evaluation of crossresistance demonstrated that no individual strain out of 198 was in parallel susceptible to all eight antifungal agents tested. Cross-resistance between azoles, echinocandins, amphotericin $B$, and flucytosine could be detected quantitatively with a new method for fungi. It ranges from two to sevenfold, and demonstrates quantitatively different and species-specific susceptibility/resistance patterns.
\end{abstract}

Keywords- hyphomycetes, azoles, echinocandins, flucytosine, amphotericin B, cross-resistance, susceptibility pattern analysis

Running Title- Antifungal susceptibility profiles in hyphomycetes

Citation: Schmalreck A.F., et al. (2014) Cross-resistance of Azoles, Echinocandins, Flucytosine and Amphotericin B in Clinically Important Hyphomycetes. International Journal of Microbiology Research, ISSN: 0975-5276 \& E-ISSN: 0975-9174, Volume 6, Issue 1, pp.-519-544.

Copyright: Copyright@2014 Schmalreck A.F., et al. This is an open-access article distributed under the terms of the Creative Commons Attribution License, which permits unrestricted use, distribution and reproduction in any medium, provided the original author and source are credited.

\section{Introduction}

Invasive fungal infections (IFI) have become increasingly common among immunocompromised patients including solid-organ or haematopoietic stem-cell transplant recipients, and of individuals who are on immunosuppressive drug regimens [1]. Although Aspergillus species are the most common causes of invasive mould infections in immunocompromised patients, and most frequently isolated as etiological agents of mycotic keratitis [2]. Emergence of rare invasive fungal diseases such as entomophtoramycosis and mucromycosis caused by Glomeromycota (Zygomycota) [3,4] as well as infections by Fusarium, Haematonectaria haematococca (F. solani), Pseudoallescheria, Scedosporium, Alternaria, Bipolaris, Cladophialophora, Sarocladium (Acremonium), Trichoderma sp. [59], and dematiaceous fungi that cause phaeohypho-mycosis [10-
13] has been reported in the last decade. Despite a general susceptibility to various antifungal compounds [14] an increase in resistance to commonly prescribed antifungal drugs, and an epidemiological shift to more drug-resistant strains with intrinsic and acquired resistance of Aspergillus species had been documented [15 $-19,28,29]$. Additionally, several species in the Aspergillus fumigatus complex appear to be resistant to azoles with evidence of in vitro and in vivo correlation [20-30]. In addition, treatment failure of Aspergillus infections [27,30-36] and emergence of azoleresistance due to agricultural azole use $[37,118,119]$ has been reported. Amphotericin B has limited activity against Aspergillus terreus and Aspergillus nidulans [38-43]. To support patient management, various antifungal susceptibility testing methods have been proposed, including agar-, macro-, and micro-dilution, disk 
diffusion, strip-, and some other commercial tests [24,27,44-46]. As drawback and according to the current recommendations the production of fungal spores is necessary in order to comply with the test requirements [51,59]. This holds true also for an improved and more rapid conidial viability assay [47]. Therefore, pre-testing times of up to 14 days, and only the susceptibility testing of sporulating fungi can be achieved. To expand the test spectrum and to fasten in vitro susceptibility testing, improved methodology is deemed necessary. In this context, routine clinical mould isolates were tested by a new inoculum preparation method [49] in two different media - Yeast Sensitivity Testing (YST) according to DIN [50], and RPMI 1640 medium according to EUCAST [51]. In order to detect quantitatively cross-resistance in moulds, which is reported to emerge significantly $[12,13,32,36,52,53,54,55]$, multi-resistance was evaluated by susceptibility pattern analysis (SPA), a new method for fungi.

\section{Materials and Methods \\ Organism Collection}

A total of 198 moulds [Table-1] were included in this study, which was performed in parallel to a recent collaborative yeast susceptibility investigation [56]. The clinical routine specimens and their origin sent to the participating clinical microbiology laboratories are given in [Table-2]. Candida parapsilosis ATCC 22019, Candida krusei ATCC 6258, and Aspergillus fumigatus ATCC 900211 accompanied each test run as quality control strains (data on file) Organism identification and differentiation were performed as described elsewhere [49].

Table 1- Number, and percentage of tested moulds $(n=198)$, listed with their reported names and current valid taxonomic status.

\begin{tabular}{|c|c|c|c|c|c|}
\hline Current valid genus/species name1) & Species names as reported & Species abbreviation ${ }^{2}$ & Isolates tested & $\%$ of species & $\%$ of total \\
\hline Absidia & Absidia corymbifera & Ab. corymbifera & 2 & 100.0 & 1.0 \\
\hline Sarocladium strictum & Acremonium strictum & Sa. strictum & 1 & 100.0 & 0.5 \\
\hline \multirow[t]{6}{*}{ Aspergillus } & Aspergillus & & 151 & 100.0 & 76.3 \\
\hline & Aspergillus fumigatus & A. fumigatus & 89 & 58.9 & 45.0 \\
\hline & Aspergillus flavus & A. flavus & 26 & 17.2 & 13.1 \\
\hline & Aspergillus niger & A. niger & 18 & 11.9 & 9.1 \\
\hline & Aspergillus terreus & A. terreus & 13 & 8.6 & 6.6 \\
\hline & Aspergillus nidulans & A. nidulans & 1 & 0.7 & 0.5 \\
\hline \multirow[t]{4}{*}{ Aspergillus } & Emericella nidulans & A. nidulans & 1 & 0.7 & 0.5 \\
\hline & Aspergillus glaucus & A. glaucus & 1 & 0.7 & 0.5 \\
\hline & Aspergillus hollandicus & A. hollandicus & 1 & 0.7 & 0.5 \\
\hline & Aspergillus sydowii & A. sydowii & 1 & 0.7 & 0.5 \\
\hline \multirow[t]{3}{*}{ Fusarium } & Fusarium & & 9 & 100.0 & 4.6 \\
\hline & Fusarium oxysporum & F. oxysporum & 6 & 66.7 & 3.0 \\
\hline & Fusarium spp. & F. spp. & 3 & 33.3 & 1.5 \\
\hline Haematonectria haematococca & Fusarium solani & H. haematococca & 8 & 100.0 & 4.0 \\
\hline \multirow[t]{2}{*}{ Mucor } & Mucor & & 4 & 100.0 & 2.0 \\
\hline & Mucor spp. & M. spp. & 4 & 100.0 & 2.2 \\
\hline Pirella circinans & Mucor circinans & P. circinans & 1 & 100.0 & 0.5 \\
\hline \multirow[t]{2}{*}{$\overline{\text { Paecilomyces }{ }^{3)}}$} & Paecilomyces spp. & Pa. spp. & 2 & 100.0 & 1.0 \\
\hline & Rhizomucor & Rm. spp. & 4 & 100.0 & 2.0 \\
\hline \multirow[t]{2}{*}{ Rhizopus } & Rhizopus & & 4 & 100.0 & 2.0 \\
\hline & Rhizopus microsporus & Rh. microsporus & 1 & 25.0 & 0.5 \\
\hline \multirow[t]{2}{*}{ Rhizopus arrhizus } & Rhizopus oryzae & Rh. arrhizus & 1 & 25.0 & 0.5 \\
\hline & Rhizopus spp. & Rh. spp. & 2 & 50.0 & 1.0 \\
\hline Scedosporium & Scedosporium & & 6 & 100.0 & 3.0 \\
\hline NB & Scedosporium prolificans & Sc. prolificans & 6 & 100.0 & 3.0 \\
\hline Pseudallescheria & & & 4 & 100.0 & 2.2 \\
\hline Pseudallescheria boydii & Scedosporium apiospermum & Ps. boydii & 4 & 100.0 & 2.2 \\
\hline Trichoderma & Trichoderma spp. & T. spp. & 2 & 100.0 & 1.0 \\
\hline
\end{tabular}

According to Index Fungorum [http://www.indexfungorum.org/Names/Names.asp]

Due to several genera with the same alphabetic letter, the listed acronyms are used in the text or tables.

As Paecilomyces was not identified to the species level, one the following species may apply instead: Acremonium, Beauvaria, Acrophialophora, ChamaeIomyces, Clonostachys, Cordyceps, Isaria, Mariannaea, Purpureocillium, Sagenomeöa, Sagrohamala, Sarcocladium, Septofusidium, Taifanglania, Verticillium

Table 2- Distribution frequency $(\mathrm{n})$ of specimens* ${ }^{*}$ per clinical speciality*

\begin{tabular}{|c|c|c|c|c|c|}
\hline (n / \%) Clinic / Ward * & Specimen* N=198 /100 & Liquids (sterile) 91 / 45.9\% & Blood Culture 18 / 9.1 & Solid Material 60 / 30.3 & Swab $29 / 14.7$ \\
\hline Anaesthetics & $7 / 3.5$ & 4 & - & - & 3 \\
\hline Dermatology & $9 / 4.6$ & - & - & 2 & 7 \\
\hline Ear, Nose, Throat & $10 / 5.1$ & - & - & - & 10 \\
\hline External Office/Ward & $74 / 37.4$ & 17 & 6 & 48 & 3 \\
\hline General Medicine & $27 / 13.6$ & 16 & 8 & 3 & - \\
\hline Oncology, Haematology & $3 / 1.5$ & 2 & - & 1 & - \\
\hline Orthopaedics & $1 / 0.5$ & - & - & 1 & - \\
\hline Pathology & $3 / 1.5$ & - & - & 1 & 2 \\
\hline Surgery & $8 / 4.0$ & 4 & - & 1 & 3 \\
\hline Transplantation & $6 / 3.0$ & 4 & 1 & 1 & - \\
\hline
\end{tabular}

*Specimens and clinical specialities comprise different varieties, which are described elsewhere [150] 


\section{Antimicrobial Susceptibility Testing}

Microdilution testing was based on a modified micro-broth dilution standard of EUCAST as described [49,58,59], with two premanufactured culture media (YST and RPMI 1640, heipha $\mathrm{GmbH}$, Germany), both containing $2 \%$ glucose (final concentration). They were tested in parallel in ready-to-use 96 well microdilution plates (Merlin $\mathrm{GmbH}$, Germany) containing the pre-prepared (freezedried) serial $\log _{2}$-dilutions of the antifungal agents (AFA): amphotericin $B(A M B)$, flucytosine (FCY), fluconazole (FLC), posaconazole (PSC), voriconazole (VRC), anidulafungin (ANF), caspofungin (CSF), and micafungin (MCF).

\section{Inoculum Preparation}

Confectioning of the inoculum was performed as already described [49], and therefore is mentioned only briefly. For rapid inoculum preparation, $10 \mathrm{ml}$ of $0.85 \%$ phosphate buffered saline plus $0.2 \mathrm{ml}$ Tween ${ }^{\circledR} 80$ were dropped onto fresh grown fungal colonies (normally $<48 \mathrm{~h}$ ) on Sabouraud-2\%-glucose (SAB) agar and were gently probed with the tip of a transfer pipette. The mycelia and conidia containing mixture was transferred to and homogenized (for approximately $40 \mathrm{sec}$ ) in a micro-homogenizer system (IKA ${ }^{\circledR}$ Tube Drive; IKA, Germany) containing 15 stainless steel balls. After the suspension was microscopically controlled, a unique inoculum of $2-5 \times 10^{4}$ viable units (vu) per $\mathrm{ml}$ was adjusted by means of a haemocytometer or (after calibration) with a photometer. Here, "vu" is defined as a countable homogenous suspension with almost uniform particles resulting in growth of single colonies when an aliquot is plated onto a solid culture medium. The final inoculum was checked by plating $100 \mu$ lonto SAB agar plates and incubating for $48 \mathrm{~h}$ at $36 \pm 1^{\circ} \mathrm{C}$, with subsequent counting of the grown colony forming units (cfu), respectively vu. Minimum inhibitory concentration (MIC) endpoint determinations with both culture media were performed visually after $24 \mathrm{~h}$ and $48 \mathrm{~h}$ of incubation at $36 \pm$ $1^{\circ} \mathrm{C}$ against the growth control. The MIC was determined as the lowest drug concentration that prevented any discernible growth (optically clear) as compared to the drug free growth control, respectively at least of $80 \%$ reduction of growth compared to the control well for not clear cut MICs. The mean-reading times for all moulds were for YST medium $29 \mathrm{~h}$ (referred as $24 \mathrm{~h}$ ) and $53 \mathrm{~h}$ (referred as $48 \mathrm{~h}$ ); and for RPMI 1640 medium $31 \mathrm{~h} \mathrm{(24} \mathrm{h)} \mathrm{and}$ $55.4 \mathrm{~h}(48 \mathrm{~h})$. FLC was on the ready to use microdilution panel for the in parallel performed collaborative study, and despite its inefficiency for moulds, it was used as quality control marker and for further cross- and parallel-resistance evaluations.

\section{Breakpoints}

Due to the lack of appropriate breakpoints, for the comparison purposes of this study, and if not otherwise indicated, low level MICs were defined as $\leq 1 \mathrm{mg} / \mathrm{l}$ (susceptible, S), and elevated or high level MICs as $>2 \mathrm{mg} / \mathrm{l}$ (resistant, R) for all antifungal agents (AFA) except PSC, where the Aspergillus breakpoints of EUCAST with $\mathrm{S} \leq 0.125 \mathrm{mg} / \mathrm{l}$ and $\mathrm{R}>0.5 \mathrm{mg} / \mathrm{l}$ were applied. The epidemiological cut-off value (ECV) was calculated according to Arendrup, et al [60], with the median MIC as basis. In addition, parallelresistance (defined as resistance among members of the same drug-class) and cross-resistance (resistance of members among different drug-classes) were evaluated by susceptibility pattern analysis [61]. The susceptibility pattern (SP) obtained by SPA, analogous to a resistance pattern of bacteria [61], was defined as the artificial sequence of the assessed MICs of the different AFA, however in a default sequential arrangement (SP-profile, e.g., SP: $\left.R_{A M B}-R_{I T R}-R_{P S C}-R_{V R C}-R_{F C Y}-R_{C S F}\right)$, where as appropriate, $R$ may be replaced by "S" or "l".

\section{Statistical Analyses}

All calculations and statistical analyses were performed with $\log _{2-}$ MIC values with $S A S^{\circledR}$ software (SAS ${ }^{\circledR}$ Institute, Cary, USA - Heidelberg, Germany). The antilog of the calculations is displayed as MIC. If not otherwise indicated, and for a better overview, percentage-values are given in round figures.

\section{Results \\ Inoculum}

With the new inoculum preparation, directly from fresh grown fungal surface cultures, a rapid, reliable inoculum could be achieved at each test-day from the freshly grown fungal surface cultures. These standardized cell suspensions yield reproducible viable units by further subcultures. That almost identical results are obtained, when tested in parallel with a conidia-inoculum according to EUCAST had already been shown in a feasibility study [49]. In addition, the possibility of mould susceptibility testing with either germinated or ungerminated conidia was demonstrated elsewhere $[62-64,83]$. As determined in the microdilution system, the new inoculum method, together with the faster growth rates in the $2 \%$ glucose supplemented test media, for all 198 filamentous fungi a susceptibility test time (without pre-culturing) of $22 \mathrm{~h}-72 \mathrm{~h}$ (mean test time for YST medium 29h (first possible endpoint determination) to $53 \mathrm{~h}$ (second reading), and for RPMI 1640 medium $31 \mathrm{~h}$ and $55 \mathrm{~h}$, respectively) was revealed. Although sometimes only a few colonies could be obtained (3-4 pre-culture plates necessary) the default inoculum of $2-5 \times 10^{4} \mathrm{vu}$ was widely achieved, i.e., sufficient homogeneous material for reproducible susceptibility testing of all 13 genera and 24 species listed in [Table-1]. Another advantage of the "new" inoculum was that like with amphotericin B, echinocandin endpoints could be clearly detected visually, i.e. without determining the minimum effective concentration (MEC) [65], which has been proposed by Kurtz, et al [66] for the endpoint determinations of lipopeptides. As already mentioned [49] and demonstrated here when applying the "new" inoculum also echinocandins endpoints can be detected with the naked eye rather to use the cumbersome MEC determinations or, alternatively by a micro-colony imaging assay [67]. Therefore, additional MEC determinations for echinocandins were not performed. This is supported as the endpoint determinations results of the 198 mould isolates and the three echinocandins, which showed for the $24 \mathrm{~h} / 48 \mathrm{~h}$ comparison an essential agreement (EA) of $93 \%-99 \%$ for YST and $94-97 \%$ for RPMI medium, and for both, the $24 \mathrm{~h}-24 \mathrm{~h}$ and $48 \mathrm{~h}-48 \mathrm{~h}$ comparison of YST and RPMI medium an EA of $88 \%-98 \%$ and $94 \%-98 \%$, respectively, was achieved [Table-3].

\section{Performance of the Culture Media}

With the media recommended by DIN (YST $+2 \%$ glucose $+0.5 \mathrm{mg}$ methylene blue/l) and EUCAST (RPMI $+2 \%$ glucose) for susceptibility testing of fungi similar MIC values for all eight AFAs were obtained with an overall endpoint concordance of $93 \%$ when the $24 \mathrm{~h}$ and $48 \mathrm{~h}$ endpoint was determined with the same medium and an $95 \%$ agreement when the $24 \mathrm{~h}$ and $48 \mathrm{~h}$ endpoints of the two different media were compared [Table-3]. The essential agreement (EA: \% of MICs of an individual AFA within \pm 1 log2-dilution) for the different AFA was $74 \%$ (AMB) to $99 \%$ (MCF) and YST medium, 
and $77 \%$ (FCY) to $97 \%$ (CSF, MCF) with RPMI medium, respectively a mean EA of $92 \%$ for each medium and the $24 \mathrm{~h}-48 \mathrm{~h}$ comparison [Table-3].

\section{Susceptibility Testing - MICs Distributions}

The overall MIC-distributions determined for all AFAs investigated at $24 \mathrm{~h}$ and $48 \mathrm{~h}$ with both media under test were almost analogous [Fig-1]. This was confirmed by determining the MIC- $\log _{2}-$ differences obtained by comparison of both, the MIC-values at the different endpoint reading times, and those from the different test media [Table-3], and as shown exemplarily, for both media and the distribution of voriconazole and posaconazole in [Fig-2], and of amphotericin B and caspofungin in [Fig-3]. As already known, the MICs at $48 \mathrm{~h}$ on the individual test media showed a tendency to be somewhat higher than at $24 \mathrm{~h}$, however, this was statistically not significant. The same holds true for the marginal higher MIC-values on YST medium when compared to RPMI [Fig-1] to Fig-3]. Another minor difference is that the MIC-ranges obtained with RPMImedium $\left(\log _{2}\right.$-MICs: $\left.-8-+7\right)$ spread more than with YST $(-7-+5)$ medium [Table-3]. Only about $30 \%$ of all isolates and all AFAs demonstrated an overall MIC of $\leq 1 \mathrm{mg} / \mathrm{l}$, and $65 \%$ of the moulds were found with a MIC of $\geq 4 \mathrm{mg} / \mathrm{l}$ [Table-3].

As susceptibility/resistance rates may only be calculated for species that have a sufficient number of isolates, for MIC- and crossresistance determinations the moulds were grouped as follows: Aspergillus spp. $\hat{=}$ total Aspergillus spp.; $\mathrm{OA} \triangleq$ other Aspergillus species (A. glaucus, A. hollandicus, A. sydowii); Glomeromycota $\hat{=}$ Absidia spp., Mucor spp., Rhizomucor spp., Rhizopus arrhizus, Rhizopus microsporus, Rhizopus spp.; Fusarium complex $\triangleq$ Fusarium oxysporum, Fusarium spp., Haematonectria haemato- cocca; $\mathrm{OM} \triangleq$ other moulds (Paecilomyces spp., Sarocladium strictum, Trichoderma spp.).

Instead of displaying tabled MIC-distributions, for both media, the percentages of MICs are listed for the most important $\log _{2}$-dilution steps (covering all ECVs/ECOFFs of the drugs) [Table-3], and as percentiles in [Table-4] and [Table-5]. Statistical analysis of the MIC distributions of the $24 \mathrm{~h}$ and $48 \mathrm{~h}$ endpoints are given in [Table4] for YST and in [Table-5] for RPMI medium, together with some characteristic MIC values (mean MIC, mode MIC, ECVs).

In [Table-6] the susceptibility/resistance (S-I-R)-rates of the MIC assessment are shown for all isolates, and in [Table-3] and [Table6 ] can be seen that with increasing incubation time (24h to $48 \mathrm{~h}$ ) the level of the individual MICs of each AFA will be reduced, respectively an increase of the individual MICs occurs. That the overall AFA MIC-distributions match quite well when both media are compared is demonstrated in [Fig-1] to [Fig-3]. [Table-4] and [Table-5] confirms these facts by statistical analysis. The differences of the AFA-activities in both media are marginal, and as shown, statistically not significant. However, for both media the resistance rates at $48 \mathrm{~h}$ endpoint reading were about $5 \%$ to $10 \%$ higher than at $24 \mathrm{~h}$ (up to $1 \log _{2}$-dilution). Effective to all tested moulds were only AMB $(\approx 90 \%)$, VRC $(\approx 78 \%)$, and to a lesser extent PSC ( $\approx 70 \%)$. Except to a few caspofungin susceptible Aspergillus isolates, the echinocandins were not inhibitory to this routine collective of clinical isolates [Table-6] and [Table-7].

When the MICs of all 8 antifungals and all isolates tested are considered, it can be demonstrated for both, that the individual and the complied antifungal agents showed partly a bi-modal distribution with distinct separation of low-level and high-level MICs [Fig-1].

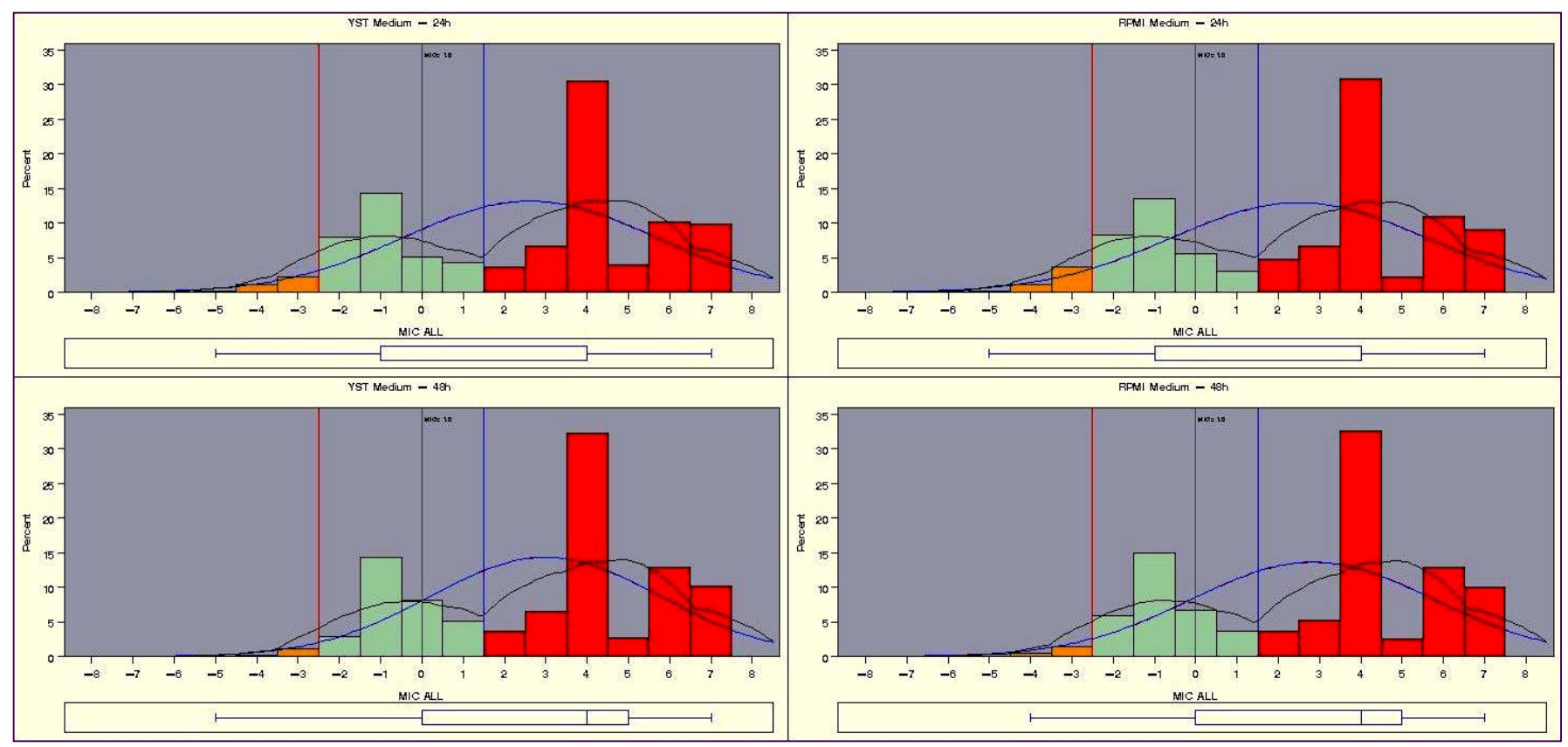

Fig. 1- Histogram of all $\log _{2}-M I C$-distribution (\% frequency) of the 8 antifungal agents in parallel tested to all fungal species ( $n=198$ ), together with their curves of normal-distribution (red), lognormal-distribution (blue), and kernel density for YST and RPMI medium , and for the two subsequent endpoint determinations (24h and $48 \mathrm{~h})$. In the schematic box-and-whisker plot in the bottom margin of the histogram, the whiskers extend to the smallest values within the lower fence and the largest value within the upper fence. Fences are defined in the terms of interquartile range (IRQ). The lower fence is $1.5 \mathrm{IRQ}$ below the first quartile and the upper fence is $1.5 \mathrm{IRQ}$ above the third quartile. Each observation outside of the fences is plotted with a symbol.

$\log _{2} / a n t i l o g(m g / l)$ explanation: $7=128 ; 6=64 ; 5=32 ; 4=16 ; 3=8 ; 2=4 ; 1=2 ; 0=1 ;-1=0.5 ;-2=0.25 ;-3=0.125 ;-4=0.063 ;-5=0.031 ;-6=0.016 ;-7=0.008 ; 8=0.004 ;$ $9=0.002 ;$ (middle vertical line $\triangleq$ MIC 1.0) 

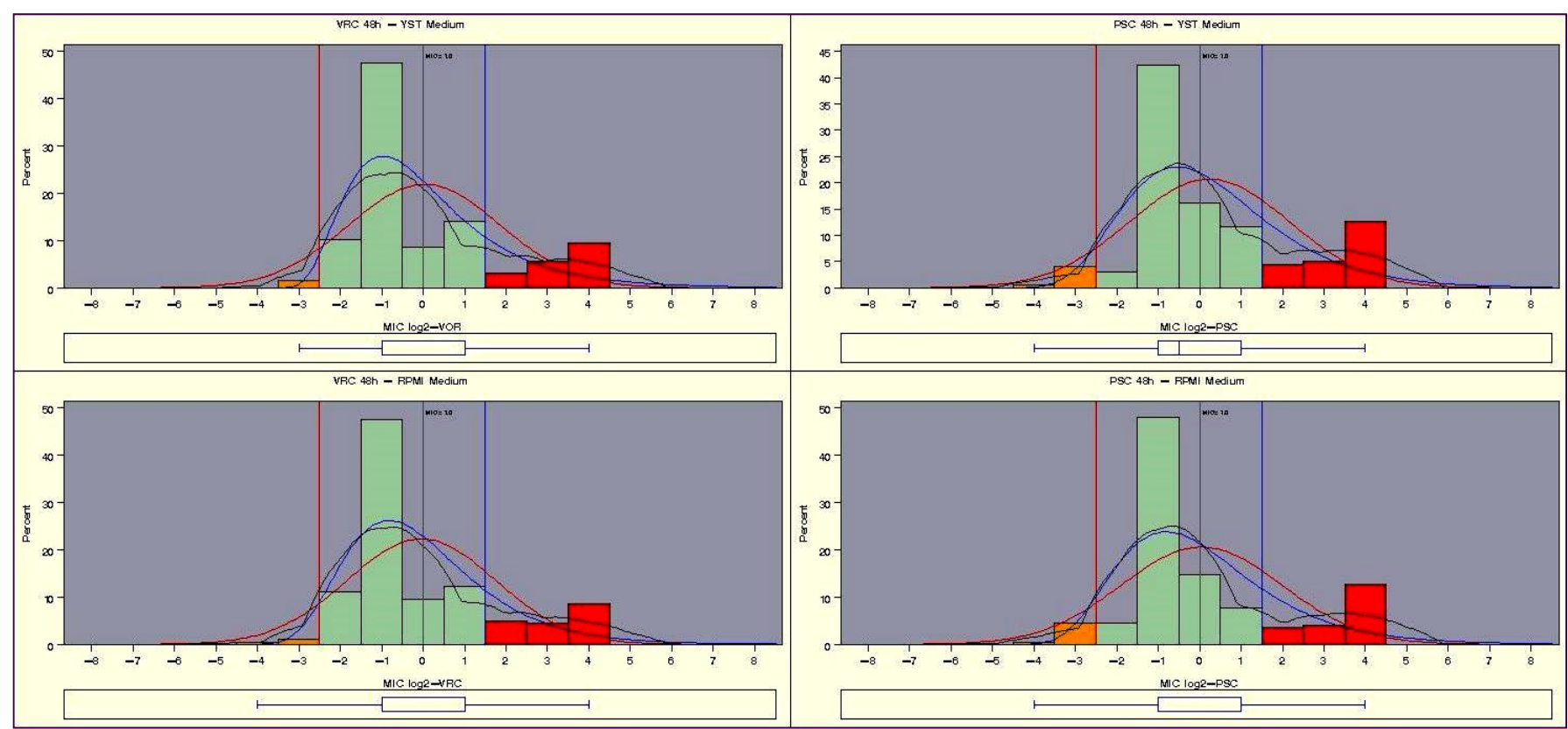

Fig. 2- Histogram of the $\log _{2}-M I C$-distribution (\% frequency) of voriconazole (VRC) and posaconazole (PSC) in parallel tested to all fungal species ( $n=198)$, together with their curves of normal-distribution (red), lognormal-distribution (blue), and kernel density for YST and RPMI medium , and for the two subsequent endpoint determinations (24h and 48h). In the schematic box-and-whisker plot in the bottom margin of the histogram, the whiskers extend to the smallest values within the lower fence and the largest value within the upper fence. Fences are defined in the terms of interquartile range (IRQ). The lower fence is $1.5 \mathrm{IRQ}$ below the first quartile and the upper fence is $1.5 \mathrm{IRQ}$ above the third quartile. Each observation outside of the fences is plotted with a symbol.

Log $_{2} /$ antilog (mg/l) explanation: $7=128 ; 6=64 ; 5=32 ; 4=16 ; 3=8 ; 2=4 ; 1=2 ; 0=1 ;-1=0.5 ;-2=0.25 ;-3=0.125 ;-4=0.063 ;-5=0.031 ;-6=0.016 ;-7=0.008 ; 8=0.004 ;$ $9=0.002 ;$ (middle vertical line $\triangleq$ MIC 1.0).
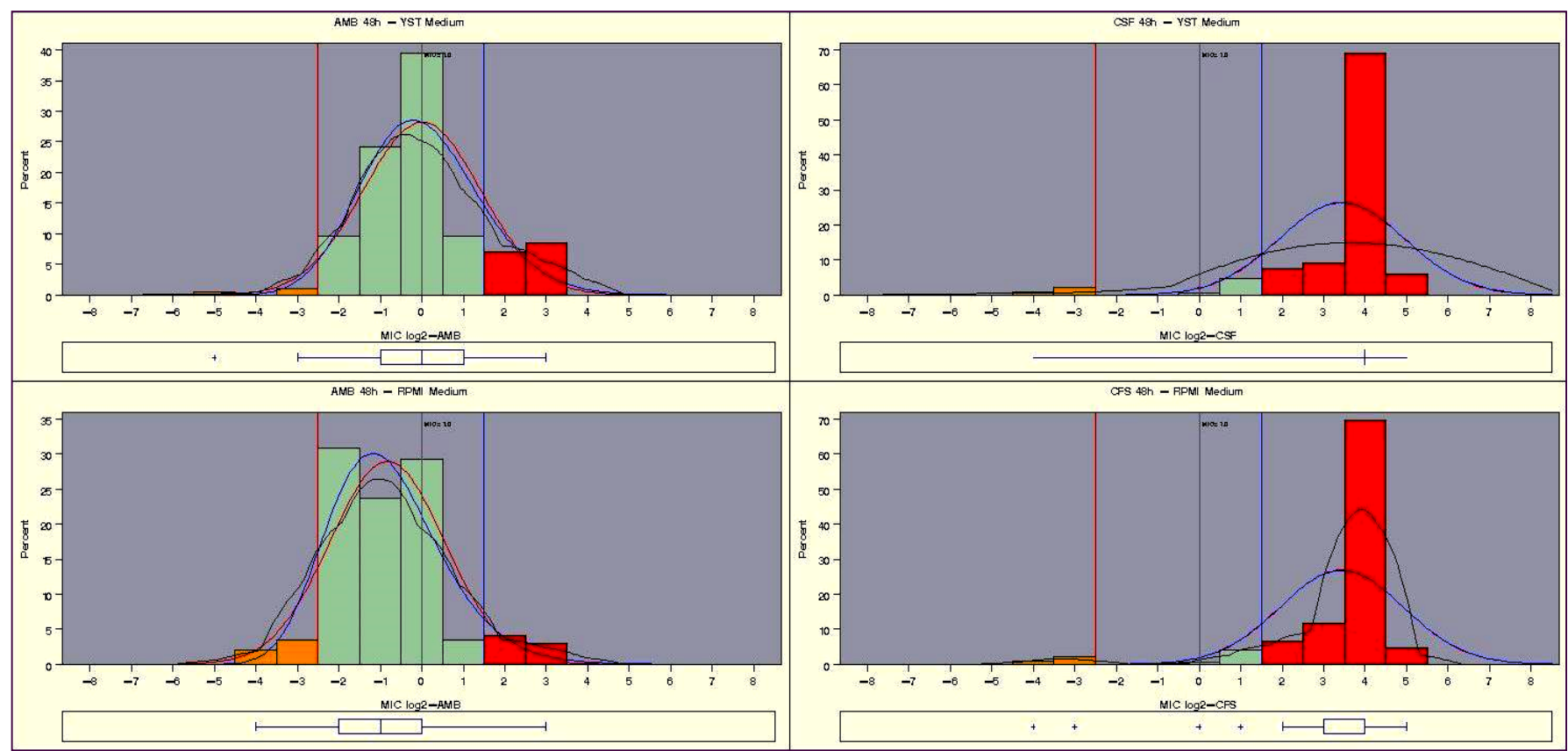

Fig. 3- Histogram of the $\log _{2}-M I C$-distribution (\% frequency) of amphotericin $B$ (AMB) and caspofungin (CSF) in parallel tested to all fungal species ( $n=198)$, together with their curves of normal-distribution (red), lognormal-distribution (blue), and kernel density for YST and RPMI medium, and for the two subsequent endpoint determinations (24h and $48 \mathrm{~h}$ ). In the schematic box-and-whisker plot in the bottom margin of the histogram, the whiskers extend to the smallest values within the lower fence and the largest value within the upper fence. Fences are defined in the terms of interquartile range (IRQ). The lower fence is $1.5 \mathrm{IRQ}$ below the first quartile and the upper fence is $1.5 \mathrm{IRQ}$ above the third quartile. Each observation outside of the fences is plotted with a symbol.

$\log _{2} /$ antilog (mg/l) explanation: 7=128; 6=64; 5=32; 4=16; 3=8; 2=4; 1=2; $0=1 ;-1=0.5 ;-2=0.25 ;-3=0.125 ;-4=0.063 ;-5=0.031 ;-6=0.016 ;-7=0.008 ; 8=0.004 ;$ $9=0.002 ;$ (middle vertical line $\triangleq$ MIC 1.0). 
The normal- and lognormal distribution of the $\log _{2}-\mathrm{MIC}$-values demonstrate that they may be antifungal agent-dependent different. As the separation point is about $1 \mathrm{mg} / \mathrm{l}$ to $2 \mathrm{mg} / \mathrm{l}$, the cut off point of $1 \mathrm{mg} / \mathrm{l}$ was chosen for further cross-resistance determinations. To check whether the "new" inoculum results are similar to data from the literature in this field, in [Table-7a] to [Table-7c] the characteristic MICs were compared (as far as available) to recent CLSI and EUCAST study data [68-77]. For the echinocandins there are similarities for visual MIC reading, however, discrepancies are to be noticed to the MEC endpoints, even within the reports claiming to use the same susceptibility testing method [Table$7 \mathrm{c}$.

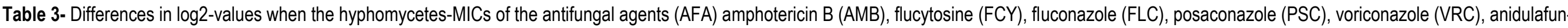
gin (ANFc), caspofungin (CSF), and micafungin (MCF) tested in YST (DIN) and RPMI (EUCST) medium are compared by the endpoint reading times (RT) at $24 \mathrm{~h}$ and $48 \mathrm{~h} .3$

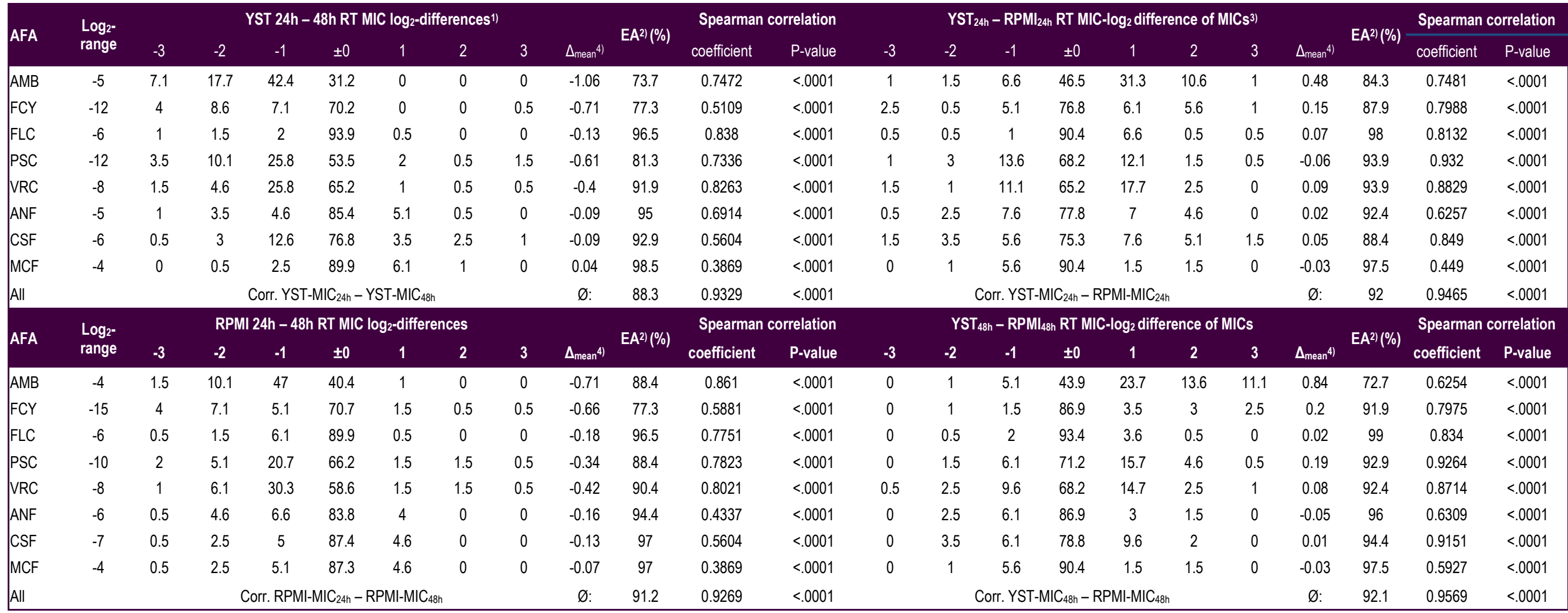

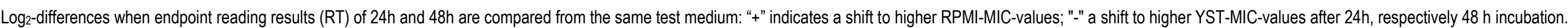
Essential agreement $(\mathrm{EA})=$ percentage of MICs within \pm one $\log _{2}$-dilution

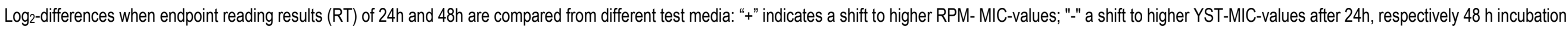
Mean of the total $\log _{2}$-differences of an individual AFA when tested on the same or with the two different media and the endpoints were read and compared after $24 \mathrm{~h}$ and $48 \mathrm{~h}$. 


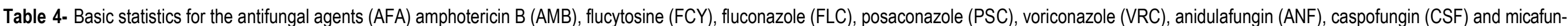

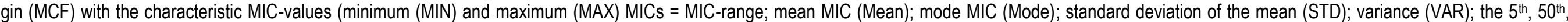

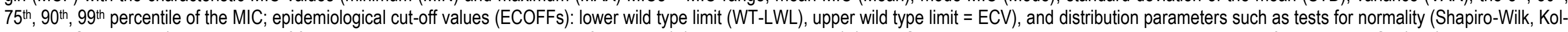

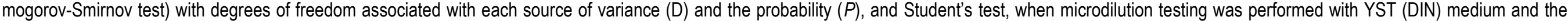
endpoint readings at $24 \mathrm{~h}$ and $48 \mathrm{~h}$.

\begin{tabular}{|c|c|c|c|c|c|c|c|c|c|c|c|c|c|c|c|c|c|c|c|c|c|c|}
\hline \multirow{2}{*}{$\begin{array}{l}\text { AFA N= } \\
198\end{array}$} & \multirow{2}{*}{$\mathrm{RT}(\mathrm{h})$} & \multicolumn{2}{|c|}{ MIC-range } & \multicolumn{2}{|c|}{ Shapiro-Wik Test } & \multicolumn{2}{|c|}{ Kolmogorov-Smirnow } & \multicolumn{2}{|c|}{ Student's t Test } & \multicolumn{2}{|c|}{ Sign Test } & \multicolumn{2}{|c|}{ Location } & \multicolumn{2}{|c|}{ Variability } & \multicolumn{2}{|l|}{ WT- } & \multicolumn{4}{|c|}{ Percentile } & \multirow{2}{*}{$\begin{array}{l}\text { ECV } \\
(\mathrm{mg} / \mathrm{l})\end{array}$} \\
\hline & & $\operatorname{MIN}(\%)(\mathrm{mg} / \mathrm{l})$ & $\operatorname{MAX}(\%)(m g / l)$ & Statistic W & $p$ Value $\operatorname{Pr}<W$ & Statistic D & $p$ Value $\mathrm{Pr}>\mathrm{D}$ & Statistic t & $\begin{array}{c}p \text { Value } P \\
\geq|t|\end{array}$ & Statistic M & $\begin{array}{l}p \text { Value } \\
P>|M|\end{array}$ & $\begin{array}{c}\text { Mean (mg/ } \\
\text { I) }\end{array}$ & $\begin{array}{l}\text { Mode } \\
(\mathrm{mg} / \mathrm{l})\end{array}$ & $\begin{array}{l}\text { STD. } \\
\text { (nxlog } 2)\end{array}$ & $\begin{array}{l}\text { VAR- } \\
\left(\log _{2}\right)\end{array}$ & $\begin{array}{c}\text { LWL (mg/ } \\
\text { I) }\end{array}$ & $\begin{array}{l}5 \% \\
(\mathrm{mg} / \mathrm{ll})\end{array}$ & $\begin{array}{l}50 \% \\
(\mathrm{mg} / \mathrm{l})\end{array}$ & $\begin{array}{l}75 \% \\
(\mathrm{mg} / \mathrm{l})\end{array}$ & $90 \%(\mathrm{mg} / \mathrm{l})$ & $99 \%(\mathrm{mg} / \mathrm{l})$ & \\
\hline \multirow{3}{*}{ AMB } & 24 & $0.031(1.0)$ & $8.0(3.0)$ & 0.925 & $<0.0001$ & 0.178 & $<0.010$ & -10.38 & $<.0001$ & -59.5 & $<.0001$ & 0.48 & 0.5 & 3 & 2 & 0.125 & 0.125 & 0.5 & 1 & 1 & 8 & 2 \\
\hline & 48 & $0.063(0.5)$ & $8.0(8.6)$ & 0.907 & $<0.0001$ & 0.248 & 0.01 & 0.05 & 0.96 & -10 & 0.0824 & 1 & 1 & 3 & 2 & 0.25 & 0.25 & 1 & 2 & 4 & 8 & 4 \\
\hline & 24 & $0.5(1.5)$ & $64.0(67.7)$ & 0.642 & $<0.0001$ & 0.396 & 0.01 & 47.75 & $<.0001$ & 95.5 & $<.0001$ & 11.16 & 64 & 3 & 3 & 16 & 2 & 64 & 64 & 64 & 64 & 256 \\
\hline \multirow{2}{*}{$\mathrm{FCY}$} & 48 & $0.5(1.0)$ & 64.0 (89.9) & 0.296 & $<0.0001$ & 0.504 & $<0.010$ & 80.29 & $<.0001$ & 96.5 & $<.0001$ & 53.16 & 64 & 1 & 2 & 16 & 16 & 64 & 64 & 64 & 64 & 256 \\
\hline & 24 & $4.0(1.0)$ & $128.0(78.3)$ & 0.489 & $<0.0001$ & 0.447 & $<0.010$ & 111.03 & $<.0001$ & 99 & $<.0001$ & 99.83 & 128 & 2 & 1 & 32 & 32 & 128 & 128 & 128 & 128 & 512 \\
\hline \multirow{2}{*}{ FLC } & 48 & $16.0(0.5)$ & $128.0(81.8)$ & 0.485 & $<0.0001$ & 0.485 & $<0.010$ & 175.55 & $<.0001$ & 99 & $<.0001$ & 108.96 & 128 & 1 & 1 & 32 & 64 & 128 & 128 & 128 & 128 & 512 \\
\hline & 24 & $0.063(2.0)$ & $16.0(12.1)$ & 0.849 & $<0.0001$ & 0.292 & $<0.010$ & -2.42 & 0.0165 & -42.5 & $<.0001$ & 0.78 & 0.5 & 2 & 4 & 0.125 & 0.125 & 0.5 & 1 & 16 & 16 & 2 \\
\hline PSC & 24 & $0.063(0.5)$ & $16.0(5.6)$ & 0.854 & $<0.0001$ & 0.284 & $<0.010$ & -2.96 & 0.0034 & -38.5 & $<.0001$ & 0.77 & 0.5 & 2 & 3 & 0.125 & 0.25 & 0.5 & 2 & 8 & 16 & 2 \\
\hline \multirow{2}{*}{ VRC } & 48 & $0.125(1.5)$ & $16.0(9.6)$ & 0.822 & $<0.0001$ & 0.305 & $<0.010$ & 0.233 & 0.8155 & -26.5 & $<.0001$ & 1.02 & 0.5 & 4 & 3 & 0.125 & 0.25 & 0.5 & 2 & 8 & 16 & 2 \\
\hline & 24 & $2.0(5.1)$ & $32.0(6.6)$ & 0.614 & $<0.0001$ & 0.437 & $<0.010$ & 63.15 & $<.0001$ & 99 & $<.0001$ & 13.43 & 16 & 1 & 1 & 4 & 2 & 16 & 16 & 16 & 32 & 64 \\
\hline \multirow{2}{*}{ ANF } & 48 & $2.0(0.5)$ & $32.0(3.5)$ & 0.559 & $<0.0001$ & 0.464 & $<0.010$ & 95.01 & $<.0001$ & 99 & $<.0001$ & 14.25 & 16 & 1 & 2 & 4 & 8 & 16 & 16 & 16 & 32 & 64 \\
\hline & 24 & $0.063(2.5)$ & $32.0(5.6)$ & 0.587 & $<0.0001$ & 0.356 & $<0.010$ & 29.79 & $<.0001$ & 92.6 & $<.0001$ & 10.21 & 16 & 2 & 3 & 4 & 2 & 16 & 16 & 16 & 32 & 64 \\
\hline \multirow{2}{*}{ CSF } & 48 & $0.063(1.0)$ & $32.0(6.1)$ & 0.571 & $<0.0001$ & 0.297 & $<0.010$ & 32.07 & $<.0001$ & 92.5 & $<.0001$ & 10.85 & 16 & 2 & 2 & 4 & 4 & 16 & 16 & 16 & 32 & 64 \\
\hline & 24 & $4.0(0.5)$ & $32.0(8.6)$ & 0.636 & $<0.0001$ & 0.421 & $<0.010$ & 105.6 & $<.0001$ & 99 & $<.0001$ & 15.32 & 16 & 1 & 1 & 4 & 8 & 16 & 16 & 16 & 32 & 64 \\
\hline MCF & 48 & $4.0(2.0)$ & $32.0(4.6)$ & 0.569 & $<0.0001$ & 0.456 & $<0.010$ & 114.87 & $<.0001$ & 99 & $<.0001$ & 18.88 & 16 & 1 & 1 & 4 & 8 & 16 & 16 & 16 & 32 & 64 \\
\hline
\end{tabular}


Table 5- Basic statistics for the antifungal agents (AFA) amphotericin B (AMB), flucytosine (FCY), fluconazole (FLC), posaconazole (PSC), voriconazole (VRC), anidulafungin (ANF), caspofungin (CSF) and micafungin (MCF) with the characteristic MIC-values (minimum (MIN) and maximum (MAX) MICs = MIC-range; mean MIC (Mean); mode MIC (Mode); standard deviation of the mean (STD); variance (VAR); the $5^{\text {th }}$, 50th $75^{\text {th }}, 90^{\text {th }}$, 99th percentile of the MIC; epidemiological cut-off values (ECOFFs): lower wild type limit (WT-LWL), upper wild type limit = ECV), and distribution parameters such as tests for normality (Shapiro-Wilk, Kolmogorov-Smirnov test) with degrees of freedom associated with each source of variance (D) and the probability (P), and Student's test, when microdilution testing was performed with RPMI 1640 (EUCAST) medium and the endpoint readings at $24 \mathrm{~h}$ and $48 \mathrm{~h}$

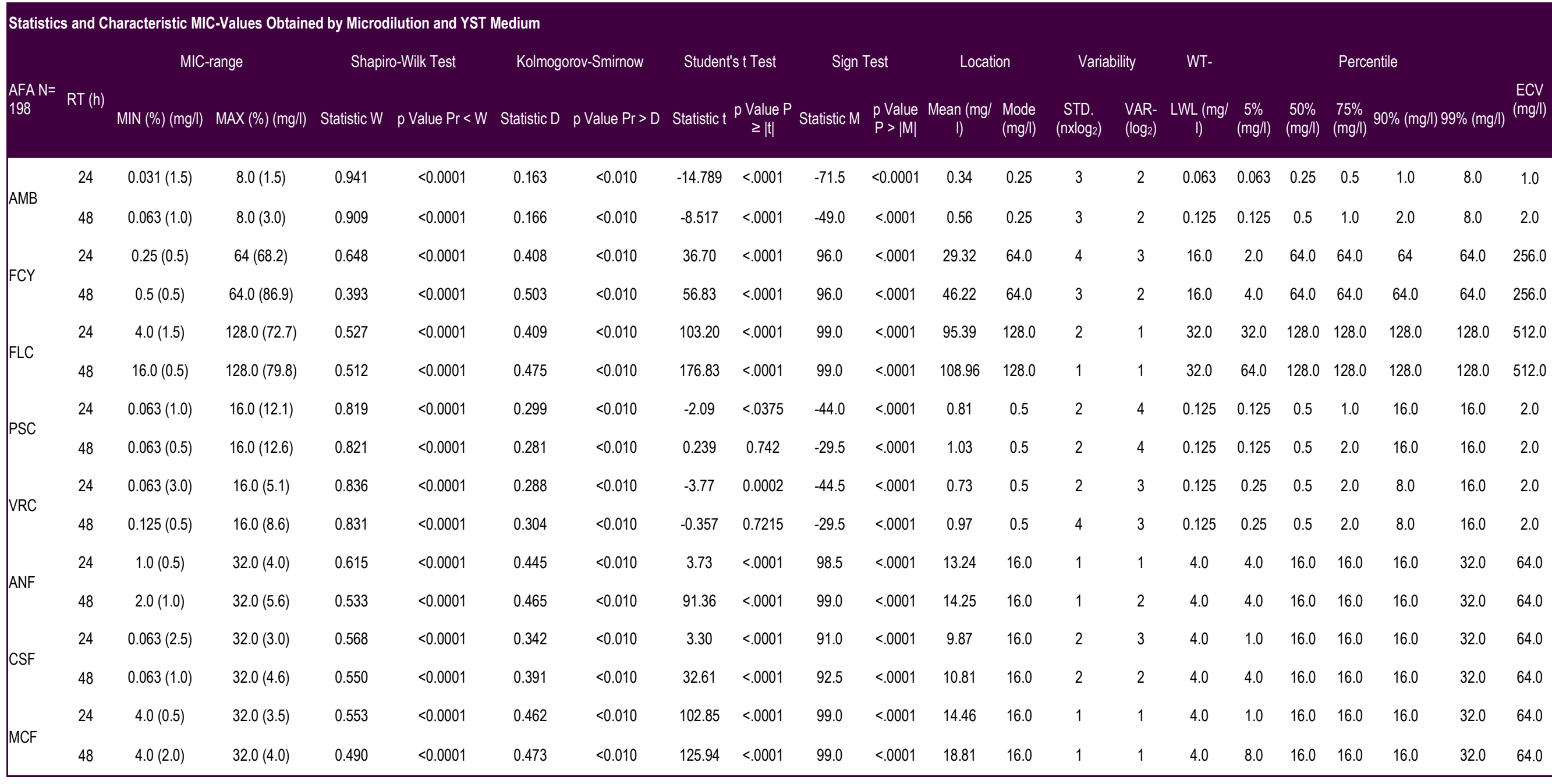

International Journal of Microbiology Research

ISSN: 0975-5276 \& E-ISSN: 0975-9174, Volume 6, Issue 1, 2014 


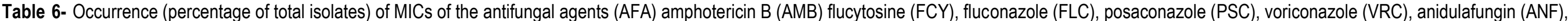

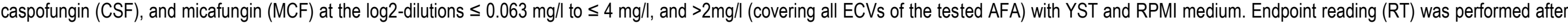
$24 \mathrm{~h}$ and $48 \mathrm{~h}$ at $36^{\circ} \mathrm{C} \pm 1^{\circ} \mathrm{C}$. The overall susceptibility/resistance was calculated for all AFA with $S \leq 1 / R>2$, except $P C S$ with $S \leq 0.25 / R>0.5$.

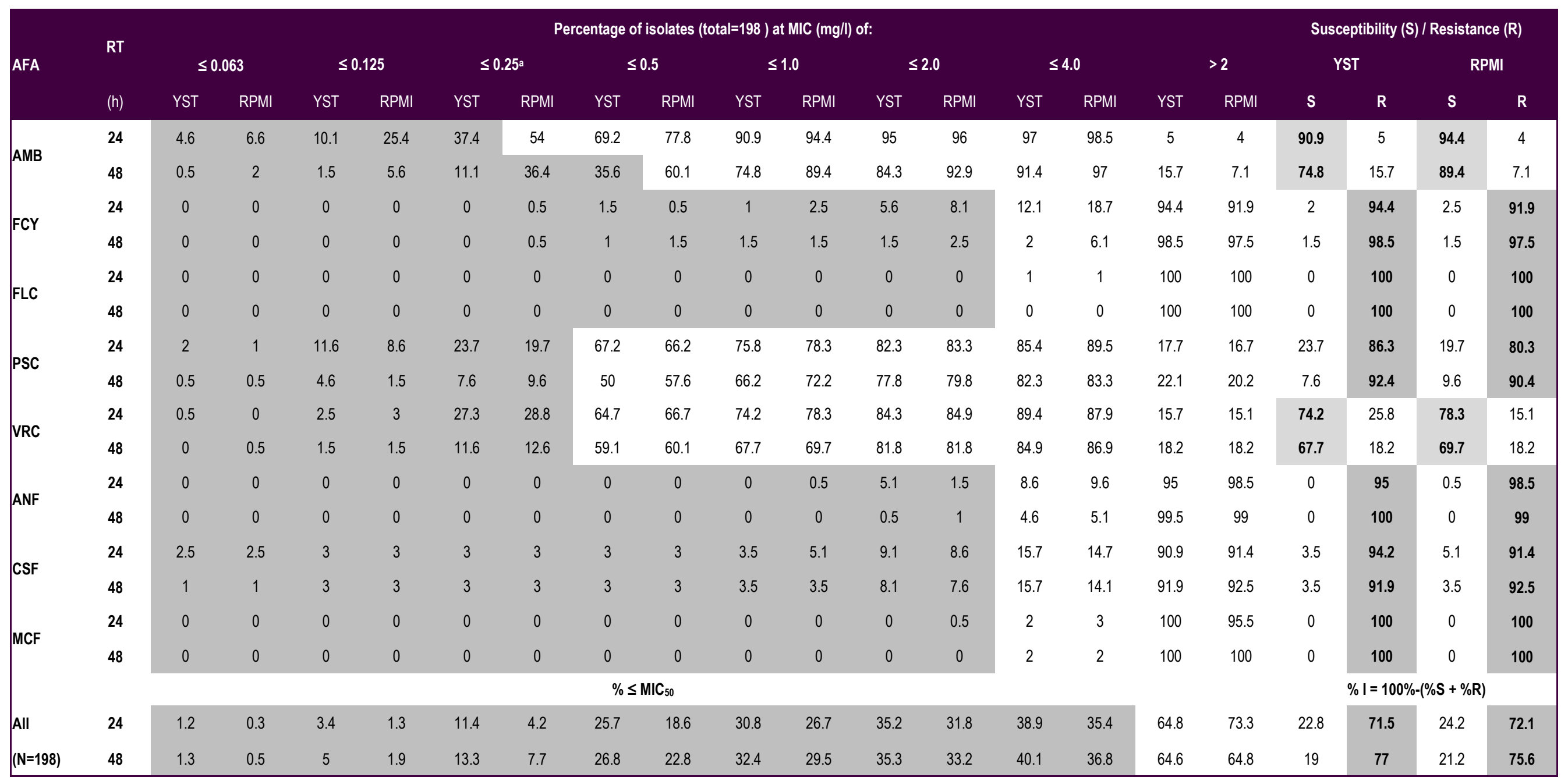

Note: In connection with in vivo mould-resistance the MIC-values of FLC are not relevant because this azole is not active in non-toxic dosage levels and therefore unsuitable for mould therapy. 
Table 7a- Comparison of species-specific characteristic MIC-values of the antifungal agents (AFA): amphotericin B (AMB), and flucytosine (FCY), with those of other studies.

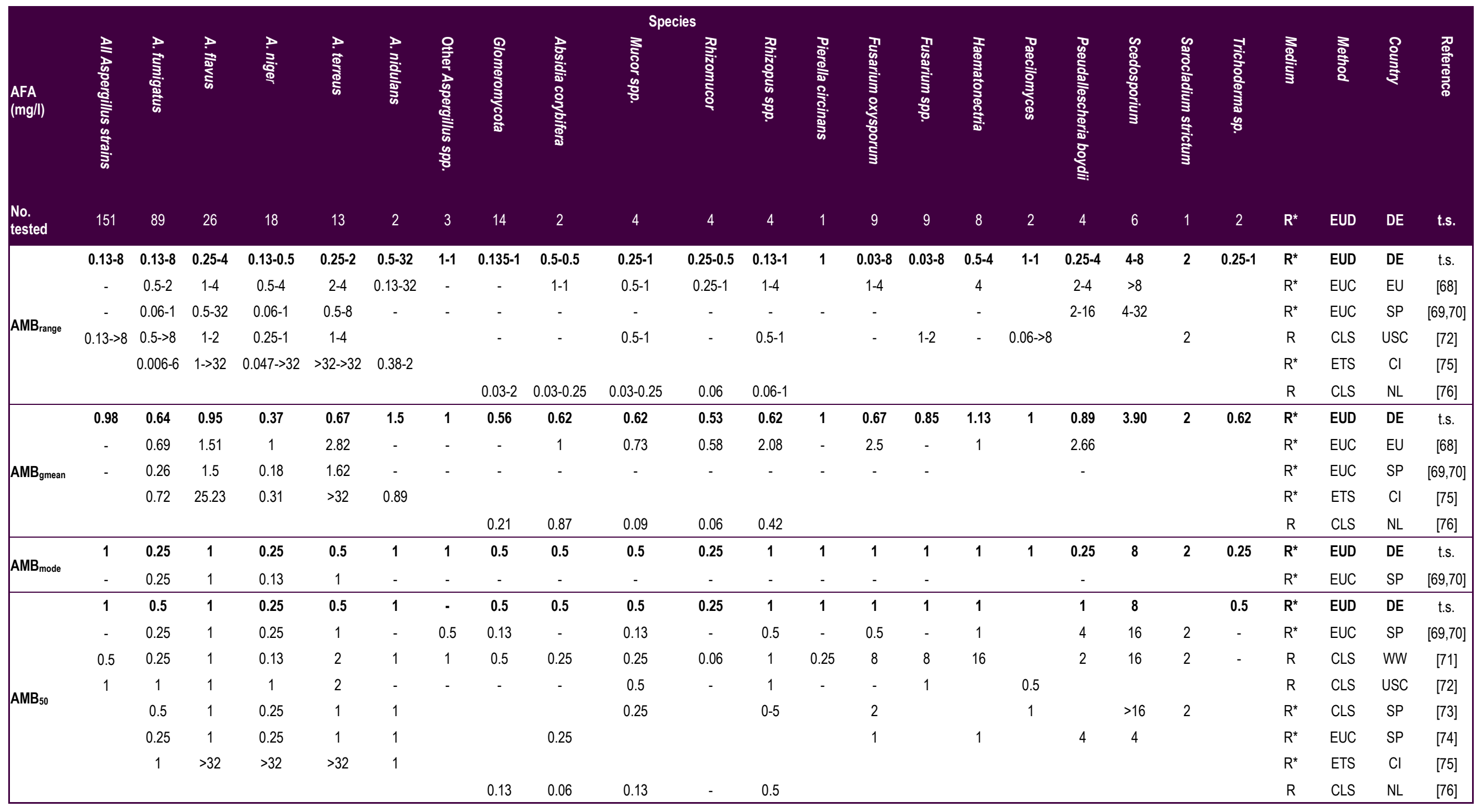


Table 7a- Continue.

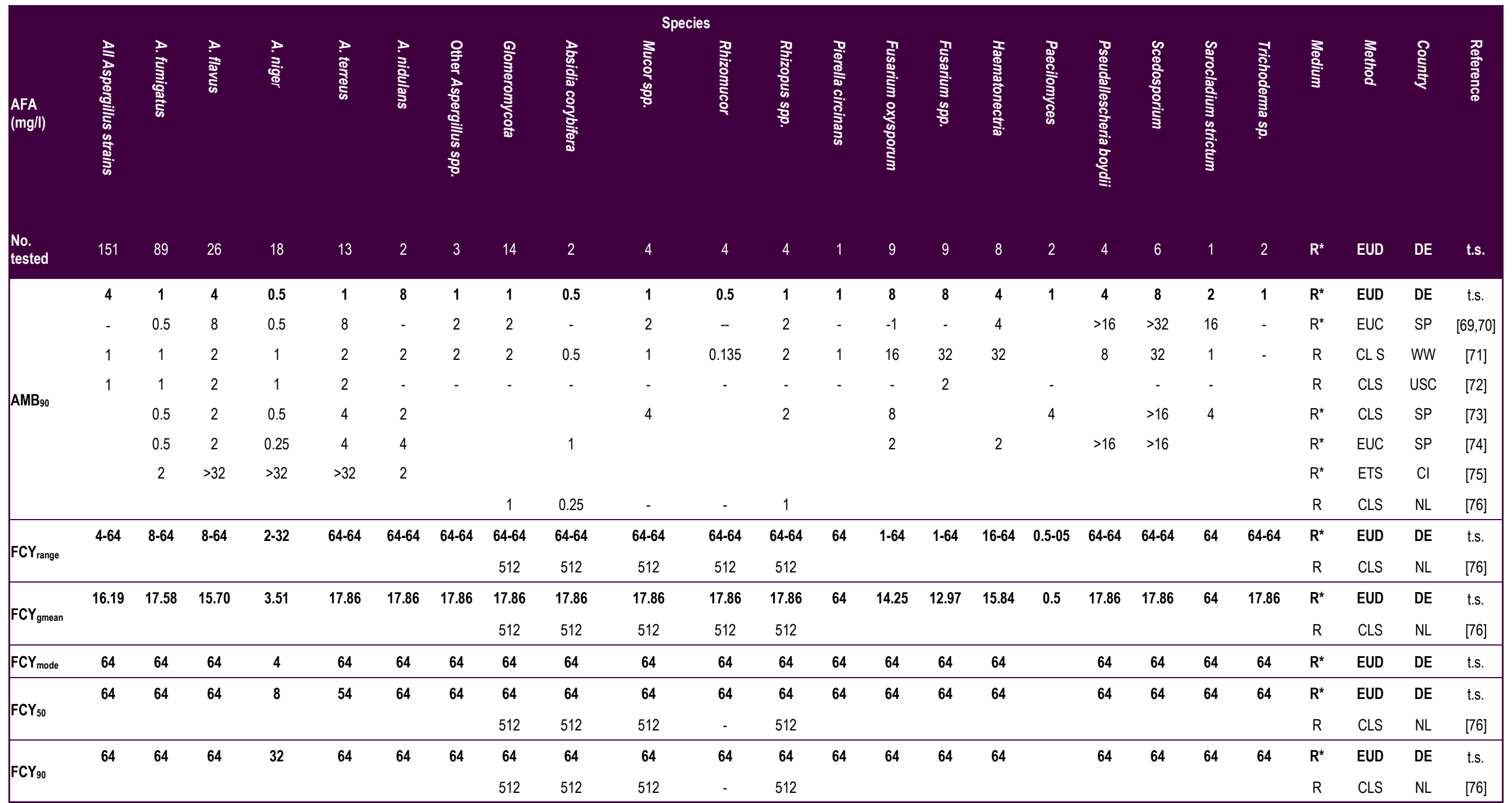

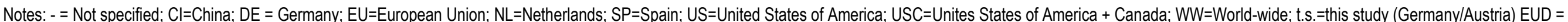

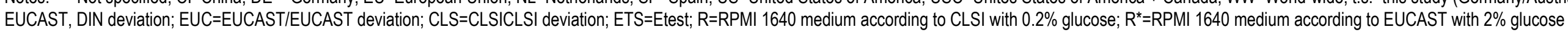


Table 7b- Comparison of species-specific characteristic MIC-values of the antifungal agents (AFA): posaconazole (PSC), and voriconazole (VRC), with those of other studies.

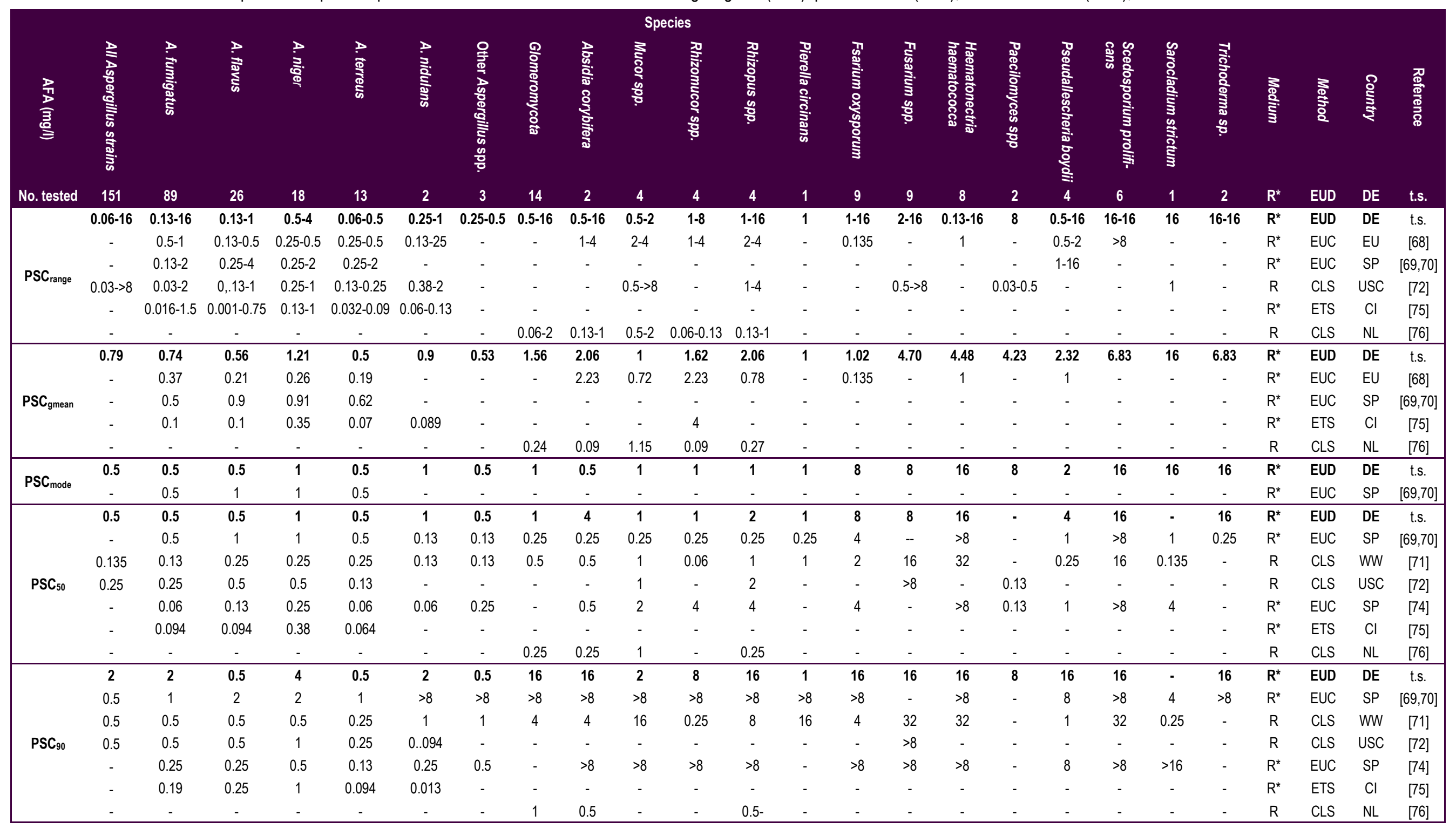


Table 7b- Continue

\begin{tabular}{|c|c|c|c|c|c|c|c|c|c|c|c|c|c|c|c|c|c|c|c|c|c|c|c|c|c|}
\hline \multirow[b]{2}{*}{ 勇 } & \multirow[b]{2}{*}{ 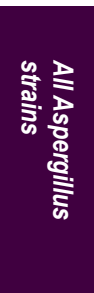 } & \multirow[b]{2}{*}{ 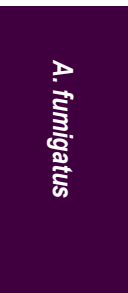 } & \multirow[b]{2}{*}{ 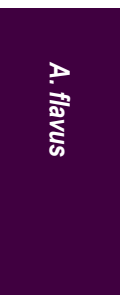 } & \multirow[b]{2}{*}{ : } & \multirow[b]{2}{*}{$\begin{array}{l}\vec{\Phi} \\
\frac{\Phi}{5} \\
\frac{\Phi}{\omega}\end{array}$} & \multirow[b]{2}{*}{ के } & \multirow[b]{2}{*}{ 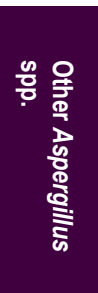 } & \multirow[b]{2}{*}{ 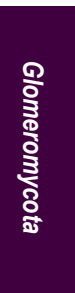 } & \multicolumn{4}{|c|}{ Species } & \multirow[b]{2}{*}{ 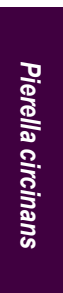 } & \multirow[b]{2}{*}{ 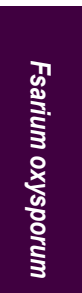 } & \multirow[b]{2}{*}{ 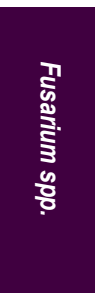 } & \multirow[b]{2}{*}{ 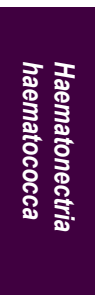 } & \multirow[b]{2}{*}{ 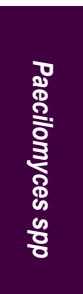 } & \multirow[b]{2}{*}{ 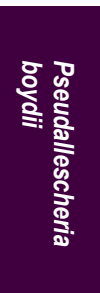 } & \multirow[b]{2}{*}{ 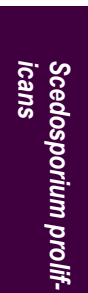 } & \multirow[b]{2}{*}{ 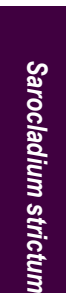 } & \multirow[b]{2}{*}{ 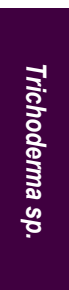 } & \multirow[b]{2}{*}{ 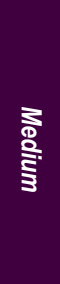 } & & & \\
\hline & & & & & & & & & 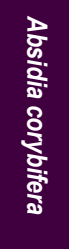 & $\begin{array}{c}\frac{8}{8} \\
\frac{8}{8} \\
\frac{0}{8} \\
\frac{0}{0}\end{array}$ & 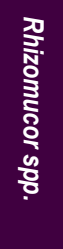 & 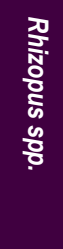 & & & & & & & & & & & $\begin{array}{l}\stackrel{8}{9} \\
\text { 홍 }\end{array}$ & $\stackrel{8}{\stackrel{8}{5}}$ & 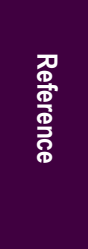 \\
\hline No. tested & 151 & 89 & 26 & 18 & 13 & 2 & 3 & 14 & 2 & 4 & 4 & 4 & 1 & 9 & 9 & 8 & 2 & 4 & 6 & 1 & 2 & $\mathrm{R}^{*}$ & EUD & $\mathrm{DE}$ & t.s. \\
\hline & $0.13-16$ & $0.25-4$ & $0.25-2$ & $0.5-4$ & $0.06-1$ & $0.13-2$ & $0.5-0.5$ & 8-16 & $8-16$ & $16-16$ & $8-16$ & $8-16$ & 16 & $0.5-16$ & $0.5-16$ & $0.5-16$ & $16-16$ & $0.25-2$ & $0.5-16$ & 8 & $2-2$ & $\mathrm{R}^{*}$ & EUD & $\mathrm{DE}$ & t.s. \\
\hline & - & $0.25-1$ & $0.5-2$ & $0.5-2$ & $0.25-1$ & $0.13-1$ & - & - & $>8$ & $>8$ & $>8$ & $>8$ & - & $>8$ & - & $>8$ & - & $2->>8$ & $>8$ & - & - & $R^{*}$ & EUC & $\mathrm{EU}$ & {$[68]$} \\
\hline & - & $0.13-2$ & $0.13-2$ & $0.25-2$ & $0.5-2$ & - & - & - & - & - & - & - & - & - & - & - & - & - & - & - & - & $\mathrm{R}^{*}$ & EUC & $\mathrm{SP}$ & {$[69,70]$} \\
\hline VRC range & $0.03->8$ & $0.06-4$ & $0.13-1$ & $0.25-2$ & $0.06-0.5$ & - & - & - & - & $1->8$ & - & $1->8$ & - & - & $0.25->8$ & - & $0.03-2$ & - & - & 8 & - & $\mathrm{R}$ & CLS & USC & [72] \\
\hline & - & $0.094-0.38$ & $0.094-0.5$ & $0.25-0.75$ & $0.25-0.5$ & $0.09-0.13$ & - & - & - & - & - & - & - & - & - & - & - & - & - & - & - & $R^{*}$ & ETS & $\mathrm{Cl}$ & [74] \\
\hline & - & - & - & - & - & - & - & $2-64$ & $2-16$ & $16-64$ & 4 & $4-64$ & - & - & - & - & - & - & - & - & - & $\mathrm{R}$ & CLS & $\mathrm{NL}$ & {$[76]$} \\
\hline & 0.72 & 0.72 & 0.54 & 1.21 & 0.51 & 0.66 & 0.62 & 5.56 & 5.37 & 6.83 & 5.37 & 4.77 & 16 & 0.98 & 2.23 & 2.46 & 8.23 & 0.79 & 3.07 & 8 & 1.62 & $\mathrm{R}^{*}$ & EUD & $\mathrm{DE}$ & t.s. \\
\hline & - & 0.36 & 0.41 & 0.33 & 0.29 & - & - & - & - & - & - & - & - & - & - & - & - & - & - & - & - & $\mathrm{R}^{*}$ & EUC & EU & [68] \\
\hline $\mathrm{VRC}_{\text {gmean }}$ & - & 0.49 & 0.66 & 0.7 & 0.92 & - & - & - & - & - & - & - & - & - & - & - & - & $0.25-16$ & - & - & - & $\mathrm{R}^{*}$ & EUC & $\mathrm{SP}$ & {$[69,70]$} \\
\hline & - & 0.18 & 0.25 & 0.38 & 0.34 & 0.11 & - & - & - & - & - & - & - & - & - & - & - & - & - & - & - & $\mathrm{R}^{*}$ & ETS & $\mathrm{Cl}$ & [75] \\
\hline & - & - & - & - & - & - & - & 11.09 & 8 & 36.76 & 4 & 8.77 & - & - & - & - & - & - & - & - & - & $\mathrm{R}$ & CLS & $\mathrm{NL}$ & [76] \\
\hline & 0.5 & 0.5 & 0.5 & 1 & 0.5 & 0.5 & 0.5 & 16 & 8 & 16 & 8 & 8 & 16 & 0.5 & 0.5 & 2 & 16 & 0.25 & 8 & 8 & 2 & $\mathbf{R}^{*}$ & EUD & $\mathrm{DE}$ & t.s. \\
\hline VRC $C_{\text {mode }}$ & - & 0.5 & 0.5 & 1 & 1 & - & - & - & - & - & - & - & - & - & - & - & - & - & - & - & - & $R^{*}$ & EUC & SP & {$[69,70]$} \\
\hline & 0.5 & 0.5 & 0.5 & 2 & 0.5 & 0.25 & 0.5 & 16 & 8 & 16 & 16 & 8 & 16 & 4 & 4 & 8 & 16 & 1 & 8 & - & 2 & $\mathrm{R}^{*}$ & EUD & $\mathrm{DE}$ & t.s. \\
\hline & - & 0.5 & 0.5 & 1 & 1 & 0.25 & 0.5 & 1 & 1 & 1 & 1 & 1 & 1 & 4 & 1 & $>8$ & - & 1 & $>8$ & 2 & 1 & $\mathrm{R}^{*}$ & EUC & $\mathrm{SP}$ & {$[69,70]$} \\
\hline & 0.25 & 0.25 & 0.5 & 0.5 & 0.25 & 0.25 & 0.25 & 16 & 16 & 64 & 2 & 16 & 64 & 4 & 16 & 16 & - & - & - & 0.25 & - & $\mathrm{R}$ & CLS & WW & [71] \\
\hline & 0.25 & 0.25 & 0.5 & 1 & 0.25 & - & - & - & - & 2 & - & 2 & $>8$ & - & 4 & - & 0.25 & - & - & - & - & $\mathrm{R}$ & CLS & USC & [72] \\
\hline $\operatorname{VRC}_{50}$ & - & 0.5 & 1 & 1 & 0.5 & 0.25 & - & - & - & $>8$ & - & $>8$ & - & 4 & - & $>8$ & - & 1 & $>8$ & 2 & - & $\mathrm{R}^{*}$ & CLS & $\mathrm{SP}$ & [73] \\
\hline & - & 0.5 & 1 & 1 & 1 & - & - & - & 0.5 & $>8$ & $>8$ & $>8$ & - & 4 & - & $>8$ & - & 0.5 & $>8$ & 4 & - & $\mathrm{R}^{*}$ & EUC & $\mathrm{SP}$ & [74] \\
\hline & - & 0.19 & 0.25 & 0.38 & 0.38 & 0.13 & - & - & - & - & - & 8 & - & - & - & - & - & - & - & - & - & $\mathrm{R}^{*}$ & ETS & $\mathrm{Cl}$ & [75] \\
\hline & - & - & - & - & - & - & - & 16 & 16 & 32 & - & - & - & - & - & - & - & - & - & - & - & $\mathrm{R}$ & CLS & $\mathrm{NL}$ & [76] \\
\hline & 2 & 2 & 0.5 & 4 & 0.5 & 1 & 0.5 & 16 & 16 & 16 & 16 & 16 & 16 & 16 & 16 & 16 & 16 & 2 & 16 & 8 & 2 & $R^{*}$ & EUD & $\mathrm{DE}$ & t.s. \\
\hline & - & 1 & 1 & 1 & 2 & $>8$ & $>8$ & $>8$ & $>8$ & $>8$ & $>8$ & $>8$ & $>8$ & $>8$ & $>8$ & $>8$ & - & 4 & $>8$ & 4 & $>8$ & $\mathrm{R}^{*}$ & EUC & $\mathrm{SP}$ & {$[69,70]$} \\
\hline & 0.5 & 0.5 & 1 & 2 & 0.5 & 1 & 1 & 128 & 128 & 128 & 16 & 128 & 128 & 32 & 32 & 32 & - & - & - & 0.5 & - & $\mathrm{R}$ & CLS & WW & [71] \\
\hline & 1 & 0.5 & 1 & 2 & 1 & - & - & - & - & - & - & - & - & - & $>8$ & - & - & - & - & - & - & $\mathrm{R}$ & CLS & USC & [72] \\
\hline VRC $_{90}$ & - & 1 & 2 & 2 & 1 & 4 & - & - & - & $>8$ & - & $>8$ & - & $>8$ & - & $>8$ & - & $>8$ & $>8$ & 4 & - & $\mathrm{R}^{*}$ & CLS & $\mathrm{SP}$ & {$[73]$} \\
\hline & - & 1 & 2 & 2 & 2 & - & - & - & $>8$ & $>8$ & $>8$ & $>8$ & - & $>8$ & - & $>8$ & - & 2 & $>8$ & 8 & - & $\mathrm{R}^{*}$ & EUC & $S P$ & [74] \\
\hline & - & 0.25 & 0.38 & 0.75 & 0.5 & 0.13 & - & - & - & - & - & - & - & - & - & - & - & - & - & - & - & $\mathrm{R}^{*}$ & ETS & $\mathrm{Cl}$ & [75] \\
\hline & - & - & - & - & - & - & - & 32 & 16 & - & - & 16 & - & - & - & - & - & - & - & - & - & $\mathrm{R}$ & CLS & $\mathrm{NL}$ & [76] \\
\hline
\end{tabular}

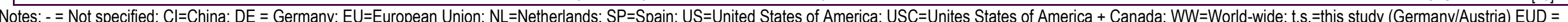

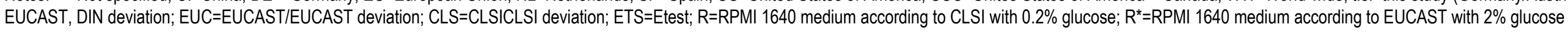

International Journal of Microbiology Research

ISSN: 0975-5276 \& E-ISSN: 0975-9174, Volume 6, Issue 1, 2014 
Table 7c- Comparison of species-specific characteristic MIC-values of the antifungal agents (AFA): anidulafungin (ANF), caspofungin (CSF), and micafungin (MCF), with those of other studies.

\begin{tabular}{|c|c|c|c|c|c|c|c|c|c|c|c|c|c|c|c|c|c|c|c|c|c|c|c|c|c|}
\hline & & & & & & & & & pecies & & & & & & & & & & & & & & & & \\
\hline $\begin{array}{l}\text { 号 } \\
\text { 章 } \\
\text { 兽 }\end{array}$ & 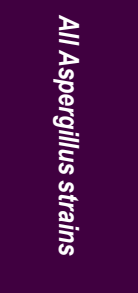 & 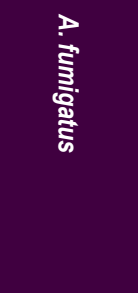 & 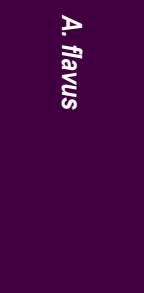 & 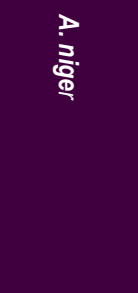 & 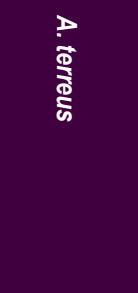 & & 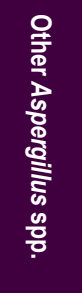 & 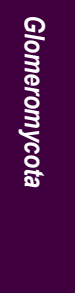 & 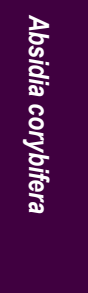 & $\begin{array}{l}\text { ș } \\
\frac{8}{8} \\
\frac{0}{8}\end{array}$ & 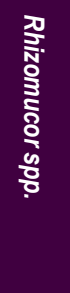 & 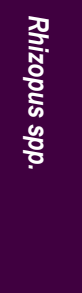 & 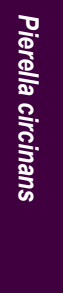 & 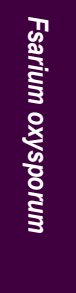 & 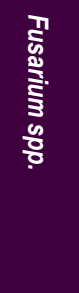 & 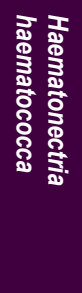 & 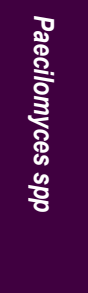 & 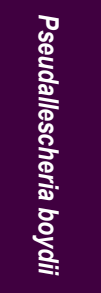 & 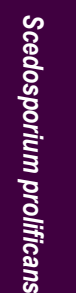 & 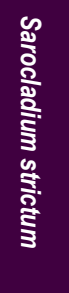 & 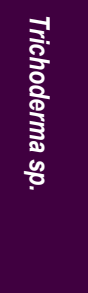 & 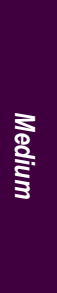 & 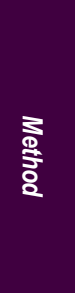 & $\frac{8}{\$}$ & 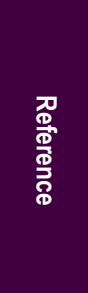 \\
\hline & $4-32$ & 4-32 & $8-32$ & 16-32 & $16-32$ & $0.03-32$ & $16-16$ & $4-16$ & $16-16$ & $16-16$ & $4-16$ & $16-16$ & 16 & 8-16 & 8-16 & 8-16 & $8-8$ & $16-32$ & $2-16$ & 16 & $16-16$ & $R^{*}$ & EUD & $\mathrm{DE}$ & t.s. \\
\hline$A N F_{\text {range }}$ & - & $0.03-0.06$ & $0.03-32$ & $0.03-0.03$ & $0.03-32$ & $0.03-0.06$ & & & & & & & & & & & & $0.25-1$ & & & & $\mathrm{R}^{*}$ & EUC & $\mathrm{SP}$ & {$[69,70]$} \\
\hline & $0.008-0.13$ & $0.008-0.13$ & $0.008-0.016$ & $0.008-0.03$ & $0.008-0.01$ & - & & & & & & & & & & & & & & & & $\mathrm{R}$ & CLS & US & {$[77]^{*}$} \\
\hline & 6.62 & 6.52 & 6.71 & 7.81 & 7.09 & 1.5 & 0.03 & 5.96 & 6.83 & 6.83 & 4.23 & 6.83 & 16 & 6.47 & 5.82 & 5.06 & 4.23 & 7.71 & 3.32 & 16 & 6.83 & $\mathrm{R}^{*}$ & EUD & $\mathrm{DE}$ & t.s. \\
\hline $\mathrm{ANF}_{\text {gmean }}$ & - & 0.03 & 1.3 & 0.03 & 0.05 & - & & & & & & & & & & & & - & & & & $\mathrm{R}^{*}$ & EUC & SP & {$[69,70]$} \\
\hline & 16 & 16 & 16 & 16 & 16 & 32 & 0.03 & 16 & 16 & 16 & 8 & 16 & 16 & 16 & 16 & 8 & 8 & 16 & 4 & & 16 & $\mathrm{R}^{*}$ & EUD & $\mathrm{DE}$ & t.s. \\
\hline $\mathrm{ANF}_{\text {mode }}$ & - & 0.03 & 32 & 0.03 & 0.03 & - & & & & & & & & & & & & - & & & & $\mathrm{R}^{*}$ & EUC & SP & {$[69,70]$} \\
\hline & 16 & 16 & 16 & 16 & 16 & 32 & 16 & 16 & 16 & 16 & 8 & 16 & 16 & 16 & 16 & 16 & 8 & 16 & 4 & 16 & 16 & $\mathbf{R}^{*}$ & EUD & $\mathrm{DE}$ & t.s. \\
\hline & - & 0.03 & $>16$ & - & 0.03 & 0.03 & 0.03 & $>16$ & $>16$ & $>16$ & $>16$ & $>16$ & & $>16$ & & $>16$ & & 0.5 & 0.06 & 0.03 & & $R^{*}$ & EUC & $\mathrm{SP}$ & [74] \\
\hline & 16 & 16 & 16 & 16 & 16 & 32 & 16 & 16 & 16 & 16 & 16 & 16 & 16 & 16 & 16 & 32 & 8 & 32 & 16 & 16 & 16 & $\mathrm{R}^{*}$ & EUD & $\overline{D E}$ & t.s. \\
\hline & - & 0.03 & 32 & 0.03 & 0.06 & - & & & & & & & & & & & & - & & & & $R^{*}$ & EUC & $\mathrm{SP}$ & {$[69,70]$} \\
\hline ANF $\left.\right|_{90}$ & 0.015 & 0.016 & 0.008 & 0.008 & 0.06 & - & & & & & & & & & & & & & & & & $\mathrm{R}$ & CLS & US & {$[77]^{*}$} \\
\hline & & 0.03 & $>16$ & - & 0.03 & 0.03 & & $>16$ & $>16$ & $>16$ & $>16$ & $>16$ & & $>16$ & & $>16$ & & 4 & $>16$ & 0.03 & & $\mathrm{R}^{*}$ & EUC & SP & [74] \\
\hline & $0.06-32$ & $0.06-32$ & $8-16$ & $8-32$ & $4-16$ & $0.25-32$ & $16-16$ & $4-16$ & $16-16$ & $16-16$ & 4-16 & $16-16$ & 16 & $2-16$ & $2-16$ & $8-32$ & 4 & $4-16$ & $2-4$ & 16 & $4-16$ & $\mathrm{R}^{*}$ & EUD & $\mathrm{DE}$ & t.s. \\
\hline & - & $0.25-1$ & $0.25-1$ & $>8$ & $0.25-1$ & $0.06-1$ & & & $>8$ & $>8$ & $>8$ & $>8$ & & $>8$ & & $>8$ & & $>8$ & $>8$ & & & $\mathrm{R}^{*}$ & EUC & EU & {$[68]$} \\
\hline & - & $0.06-2$ & $0.25-32$ & $0.06-1$ & $0.13-32$ & - & & & & & & & & & & & & $0.5-16$ & & & & $\mathrm{R}^{*}$ & EUC & $\mathrm{SP}$ & {$[69,70]$} \\
\hline $\mathrm{CSF}_{\text {range }}$ & $0.02-4$ & $0.02-2$ & $0.02-0.13$ & $0.02-0.06$ & $0.02-0.13$ & - & . & - & - & $>8->8$ & & & & & & & $0.03-8$ & & & & & $\mathrm{R}$ & CLS & WW & [71] \\
\hline & $0.008-4$ & $0.016-1$ & $0.008-0.03$ & $0.016-0.5$ & $0.016-1$ & - & & & & & & & & & & & & & & & & $\mathrm{R}$ & CLS & US & {$[77]^{*}$} \\
\hline & - & $0.032-0.13$ & $0.016-0.19$ & $0.023-0.19$ & $0.016-0.19$ & $0.05-0.09$ & & & & & & & & & & & & & & & & $\mathrm{R}^{*}$ & ETS & $\mathrm{Cl}$ & [75] \\
\hline & 5.47 & 4.73 & 6.23 & 6.83 & 6.12 & 2.7 & 6.83 & 6.38 & 6.83 & 6.83 & 5.37 & 6.83 & 16 & 5.21 & 4.23 & 5.71 & 2.61 & 3.32 & 2.23 & 16 & 4.23 & $\mathrm{R}^{*}$ & EUD & $\mathrm{DE}$ & t.s. \\
\hline & - & 0.45 & 0.28 & - & 0.19 & - & & & - & & & & & - & & & & - & & & & $R^{*}$ & EUC & EU & {$[68]$} \\
\hline $\mathrm{CSF}_{\text {gmean }}$ & - & 0.36 & 2.7 & 0.36 & 1 & - & & & & & & & & & & & & - & & & & $R^{*}$ & EUC & $\mathrm{SP}$ & {$[69,70]$} \\
\hline & - & 0.07 & 0.042 & 0.08 & 0.06 & 0.06 & & & & & & & & & & & & & & & & $\mathrm{R}^{*}$ & ETS & $\mathrm{Cl}$ & [75] \\
\hline & 16 & 16 & 16 & 16 & 16 & 32 & 16 & 16 & 16 & 16 & 16 & 16 & 16 & 16 & 16 & 8 & 4 & 4 & 4 & 16 & 16 & $\mathbf{R}^{*}$ & EUD & $\mathrm{DE}$ & t.s. \\
\hline CSF & - & 0.25 & 32 & 0.5 & 2 & - & & $>16$ & & & & & & & & & & - & & & & $\mathrm{R}^{*}$ & EUC & $S P$ & {$[69,70]$} \\
\hline
\end{tabular}

International Journal of Microbiology Research

ISSN: 0975-5276 \& E-ISSN: 0975-9174, Volume 6, Issue 1, 2014 
Table 7c- Continue.

\begin{tabular}{|c|c|c|c|c|c|c|c|c|c|c|c|c|c|c|c|c|c|c|c|c|c|c|c|c|c|}
\hline & & & & & & & & & Species & & & & & & & & & & & & & & & & \\
\hline $\begin{array}{l}\text { 苓 } \\
\text { 念 } \\
\text { 总 }\end{array}$ & 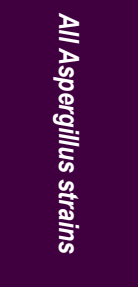 & 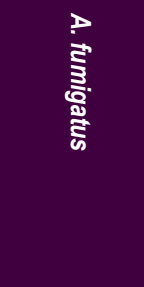 & 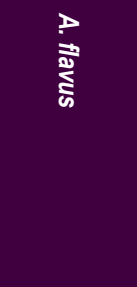 & 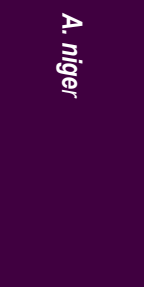 & $\begin{array}{l}\vec{\Phi} \\
\frac{\Phi}{\tilde{\omega}} \\
\frac{\Phi}{\omega}\end{array}$ & 离 & 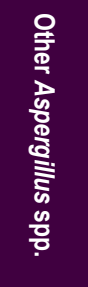 & 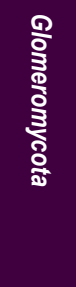 & 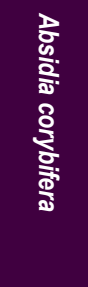 & $\begin{array}{l}\text { ș } \\
\stackrel{8}{8} \\
\frac{0}{8} \\
\frac{8}{0}\end{array}$ & 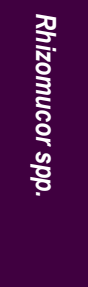 & 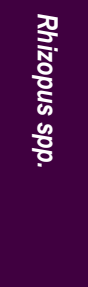 & 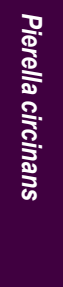 & 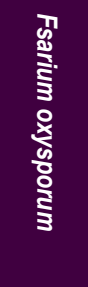 & 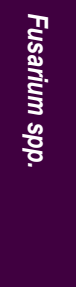 & 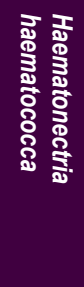 & 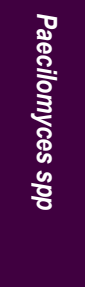 & 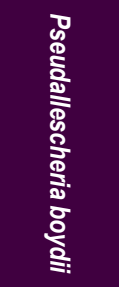 & 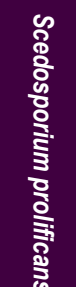 & 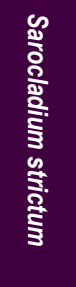 & 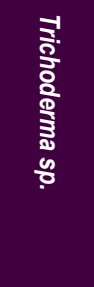 & 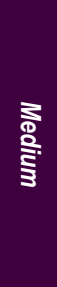 & 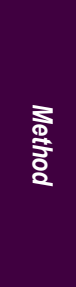 & $\stackrel{§}{\stackrel{\Xi}{5}}$ & 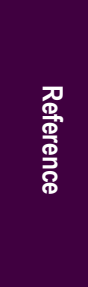 \\
\hline No. tested & 151 & 89 & 26 & 18 & 13 & 2 & 3 & 14 & 2 & 4 & 4 & 4 & 1 & 9 & 9 & 8 & 2 & 4 & 6 & 1 & 2 & $\mathbf{R}^{*}$ & EUD & DE & t.s. \\
\hline & 16 & 16 & 16 & 16 & 16 & 32 & 16 & 16 & 16 & 16 & 16 & 16 & 16 & 16 & 16 & 16 & 4 & 4 & 16 & 16 & 16 & $\mathrm{R}^{*}$ & EUD & $\mathrm{DE}$ & t.s. \\
\hline & - & 0.25 & 1 & 0.5 & 1 & - & & & & & & & & & & & & - & & & & $\mathrm{R}^{*}$ & EUC & $\mathrm{SP}$ & {$[69,70]$} \\
\hline $\mathrm{CSF}_{-}$ & 0.03 & 0.03 & 0.03 & 0.03 & 0.03 & - & - & - & - & $>8$ & - & $>8$ & - & - & $>8$ & - & 0.06 & & & & & $\mathrm{R}$ & CLS & WW & [71] \\
\hline CSS ${ }_{50}$ & 0.03 & 0.03 & 0.03 & 0.03 & 0.03 & - & & & & & & & & & & & & & & & & $\mathrm{R}$ & CLS & US & {$[77]^{*}$} \\
\hline & - & 0.25 & $>16$ & - & 0.25 & 1 & & & $>16$ & $>16$ & $>16$ & $>16$ & & $>16$ & & $>16$ & & 2 & 8 & 0.5 & & $\mathrm{R}^{*}$ & EUC & SP & [74] \\
\hline & - & 0.094 & 0.047 & 0.13 & 0.064 & 0.064 & & & & & & & & & & & & & & & & $\mathrm{R}^{*}$ & ETS & $\mathrm{Cl}$ & [75] \\
\hline & 16 & 16 & 16 & 16 & 16 & 32 & 16 & 16 & 16 & 16 & 16 & 16 & 16 & 16 & 16 & 16 & 4 & 8 & 4 & 16 & 16 & $\mathrm{R}^{*}$ & EUD & $\mathrm{DE}$ & t.s. \\
\hline & 0.06 & 1 & 32 & 1 & 4 & - & & & & & & & & & & & & - & & & & $\mathrm{R}^{*}$ & EUC & $\mathrm{SP}$ & {$[69,70]$} \\
\hline & 0.06 & 0.06 & 0.06 & 0.06 & 0.06 & - & - & - & - & - & - & & - & - & - & - & - & - & - & - & - & $\mathrm{R}$ & CLS & WW & [71] \\
\hline $\mathrm{CSF}_{90}$ & - & 0.06 & 0.03 & 0.03 & 0.5 & - & & & & & & & & & & & & & & & & $\mathrm{R}$ & CLS & US & {$[77]^{*}$} \\
\hline & - & 0.5 & $>16$ & - & 0.5 & 2 & & & $>16$ & $>16$ & $>16$ & $>16$ & & $>16$ & & $>16$ & & $>16$ & $>16$ & 1 & & $\mathrm{R}^{*}$ & EUC & $\mathrm{SP}$ & [74] \\
\hline & - & 0.094 & 0.094 & 0.19 & 0.19 & 0.094 & & & & & & & & & & & & & & & & $\mathrm{R}^{*}$ & ETS & $\mathrm{Cl}$ & {$[75\}$} \\
\hline & $4-32$ & $4-32$ & $8-32$ & $8-32$ & $16-32$ & $0.03-32$ & $\begin{array}{l}16-16 \\
\end{array}$ & $16-16$ & 16-16 & 16-16 & 16-16 & $16-16$ & 16 & $8-16$ & 8-16 & $8-32$ & 16-16 & $16-16$ & $8-16$ & 16 & 16-16 & $\mathrm{R}^{*}$ & EUD & $\mathrm{DE}$ & t.s. \\
\hline MCF range & - & $0.03-0.5$ & 0.03-32 & $0.03-0.25$ & 0.03-32 & $0.03-0.5$ & & & & & & & & & & & & $0.13-0.25$ & & & & $\mathrm{R}^{*}$ & EUC & $\mathrm{SP}$ & {$[69,70]$} \\
\hline & $0.008-0.13$ & $0.008-0.06$ & $0.008-0.03$ & $0.008-0.03$ & $0.008-0.03$ & & & & & & & & & & & & & & & & & $\mathrm{R}$ & CLS & US & {$[77]^{\star}$} \\
\hline & 6.56 & 6.55 & 6.71 & 6.53 & 7.36 & 1.5 & 6.83 & 6.83 & 6.83 & 6.83 & 6.83 & 6.83 & 16 & 6.59 & 5.82 & 5.71 & 6.83 & 6.83 & 5.82 & 16 & 6.83 & $\mathrm{R}^{*}$ & EUD & $\mathrm{DE}$ & t.s. \\
\hline $\mathrm{MCF}_{\text {gmean }}$ & - & 0.03 & 1.5 & 0.04 & 0.04 & - & & & & & & & & & & & & - & & & & $\mathrm{R}^{*}$ & EUC & $\mathrm{SP}$ & {$[69,70]$} \\
\hline & 16 & 16 & 16 & 16 & 16 & 32 & 16 & 16 & 16 & 16 & 16 & 16 & 16 & 16 & 16 & 8 & 16 & 16 & 16 & 16 & 16 & $\mathrm{R}^{*}$ & EUD & $\mathrm{DE}$ & t.s. \\
\hline $\mathrm{MCF}_{\text {mode }}$ & - & 0.03 & 32 & 0.03 & 0.4 & - & & & & & & & & & & & & - & & & & $R^{*}$ & EUC & $\mathrm{SP}$ & {$[69,70]$} \\
\hline & 16 & 16 & 16 & 16 & 16 & 32 & 16 & 16 & 16 & 16 & 16 & 16 & 16 & 16 & 16 & 16 & 16 & 16 & 16 & 16 & 16 & $\mathrm{R}^{*}$ & EUD & $\mathrm{DE}$ & t.s. \\
\hline & - & 0.03 & 32 & 0.03 & 0.03 & & & & & & & & & & & & & - & & & & $\mathrm{R}^{*}$ & EUC & $\mathrm{SP}$ & {$[69,70]$} \\
\hline $\mathrm{MCF}_{50}$ & - & 0.03 & $>16$ & - & 0.03 & & & & & & & & & & & & & & & & & $\mathrm{R}^{*}$ & EUC & $S P$ & {$[74]$} \\
\hline & 0.008 & 0.008 & 0.008 & 0.008 & 0.008 & 0.03 & 0.03 & & $>16$ & $>16$ & $>16$ & $>16$ & & $>16$ & & $>16$ & 16 & 0.03 & 8 & 0.13 & & $\mathrm{R}$ & CLS & US & {$[77]^{*}$} \\
\hline & 16 & 16 & 16 & 16 & 16 & 32 & 16 & 16 & 16 & 16 & 16 & 16 & 16 & 16 & 16 & 16 & 16 & 16 & 16 & 16 & 16 & $\mathrm{R}^{*}$ & EUD & $\mathrm{DE}$ & t.s. \\
\hline & - & 0.6 & 32 & 0.13 & 0.06 & - & & & & & & & & & & & & - & & & & $\mathrm{R}^{*}$ & EUC & $\mathrm{SP}$ & {$[69,70]$} \\
\hline $\mathrm{MCF}_{90}$ & 0.016 & 0.008 & 0.016 & 0.008 & 0.03 & - & & & & & & & & & & & & & & & & $\mathrm{R}$ & CLS & US & {$[77]^{\star}$} \\
\hline & - & 0.03 & $>16$ & - & 0.06 & 0.03 & 0.03 & & $>16$ & $>16$ & $>16$ & $>16$ & & $>16$ & & $>16$ & & $>16$ & $>16$ & 0.24 & & $\mathrm{R}^{*}$ & EUC & $\mathrm{SP}$ & [74] \\
\hline
\end{tabular}

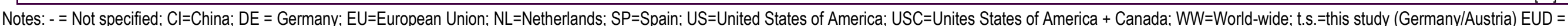

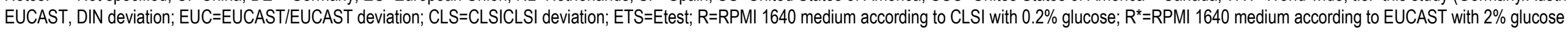

International Journal of Microbiology Research

ISSN: 0975-5276 \& E-ISSN: 0975-9174, Volume 6, Issue 1, 2014 
Table 8- Number ( $n$ ) and percentage (\%) of individual susceptibility patterns (SP) obtained by SPA from categorized antifungal agents (AFA) of amphotericin B (AMB), flucytosine (FCY), fluconazole (FLC), posaconazole (PSC), voriconazole (VRC), anidulafungin (ANF), caspofungin (CSF), and micafungin (MCF), when susceptibility testing was performed in parallel with YST and RPMI medium and endpoint reading performed at $24 \mathrm{~h}$ and $48 \mathrm{~h}$. For better readability susceptible (S) is displayed as "1", intermediate as "2", and resistance as "3" (3-leg assessment) instead of "S", "I", and "R". The SPs in the grey shadowed area on the right are derived from the SPA where the MIC assessment revealed by altered breakpoints only "S" and "R" (2-leg assessment, no intermediate tested isolates). Individual SP-profiles, which occurred in both MIC assessments (2-leg and 3-leg) are indicated with a continuous dark-grey bar.

\begin{tabular}{|c|c|c|c|c|c|c|c|c|c|c|c|c|c|c|c|c|c|c|}
\hline \multirow{5}{*}{$\begin{array}{l}\text { Susceptibility } \\
\text { Pattern } \\
\text { No. }\end{array}$} & \multicolumn{18}{|c|}{ AFA-Sequence evaluated by SPA: FCY-AMB-PCS-VRC-FLC-ANF-CSF-MCF } \\
\hline & \multicolumn{12}{|c|}{ Breakpoints: $S \leq 1, \mathrm{R}>2$; PSC: $\leq 0.125, \mathrm{R}>0.25$} & \multicolumn{6}{|c|}{ Breakpoints: $S \leq 1, R>1$; PSC: $S \leq 0.25, R>0.25$} \\
\hline & \multicolumn{6}{|c|}{ SP-profile YST Medium } & \multicolumn{5}{|c|}{ SP-profile RPMI Medium } & \multirow[b]{3}{*}{$\%$} & \multirow{2}{*}{\multicolumn{3}{|c|}{$\begin{array}{l}\text { SP-profile YST Medium } \\
48 \mathrm{~h}\end{array}$}} & SP-profile & RPMI & edium \\
\hline & 2 & & & 48 & th & & 24 & th & & 48 & & & & & & 2 & 48h & (20 \\
\hline & SP & $\mathrm{n}$ & $\%$ & SP & $\mathrm{n}$ & $\%$ & SP & $\mathrm{n}$ & $\%$ & SP & $\mathrm{n}$ & & SP & $\mathrm{n}$ & $\%$ & SP & $\mathrm{n}$ & $\%$ \\
\hline 1 & 11113333 & 1 & 0.5 & & & & & & & & & & & & & & & \\
\hline 2 & 11213333 & 2 & 1 & & & & & & & & & & & & & & & \\
\hline 3 & & & & & & & 11313313 & 2 & 1 & & & & & & & & & \\
\hline 4 & 11313333 & 1 & 0.5 & & & & 11313333 & 3 & 1.5 & & & & & & & & & \\
\hline 5 & & & & 11323333 & 1 & 0.5 & & & & & & & & & & & & \\
\hline 6 & & & & 11333333 & 2 & 1 & & & & 11333333 & 3 & 1.5 & 11333333 & 3 & 1.5 & 11333333 & 3 & 1.5 \\
\hline 7 & 21113333 & 1 & 0.5 & & & & & & & & & & & & & & & \\
\hline 8 & 21213333 & 4 & 2 & & & & 21213333 & 1 & 0.5 & & & & & & & & & \\
\hline 9 & 21223333 & 1 & 0.5 & & & & & & & & & & & & & & & \\
\hline 10 & & & & & & & 21313333 & 10 & 5.1 & 21313333 & 1 & 0.5 & & & & & & \\
\hline 11 & 21323333 & 1 & 0.5 & & & & & & & & & & & & & & & \\
\hline 12 & & & & & & & & & & 21333333 & 1 & 0.5 & & & & & & \\
\hline 13 & 31113313 & 4 & 2 & 31113313 & 2 & 1 & 31113313 & 4 & 2 & 31113313 & 2 & 1 & 31113313 & 3 & 1.5 & 31113313 & 3 & 1.5 \\
\hline 14 & 31113333 & 17 & 8.6 & 31113333 & 6 & 3.1 & 31113333 & 13 & 6.6 & 31113333 & 7 & 3.6 & 31113333 & 11 & 5.6 & 31113333 & 15 & 7.6 \\
\hline 15 & & & & 31133333 & 1 & 0.5 & & & & & & & 31133333 & 1 & 0.5 & & & \\
\hline 16 & & & & 31213313 & 3 & 1.5 & & & & 31213313 & 1 & 0.5 & & & & & & \\
\hline 17 & & & & & & & 31213133 & 1 & 0.5 & & & & & & & & & \\
\hline 18 & 31213233 & 1 & 0.5 & & & & & & & & & & & & & & & \\
\hline 19 & 31213313 & 2 & 1 & & & & & & & & & & & & & & & \\
\hline 20 & 31213333 & 87 & 44 & 31213333 & 72 & 36.4 & 31213333 & 20 & 10.1 & 31213333 & 8 & 4.1 & & & & & & \\
\hline 21 & 31223333 & 2 & 1 & 31223333 & 1 & 0.5 & & & & & & & & & & & & \\
\hline 22 & 31233233 & 1 & 0.5 & 31233233 & 3 & 1.5 & & & & & & & & & & & & \\
\hline 23 & 31233333 & 7 & 3.5 & & & & & & & & & & & & & & & \\
\hline 24 & 31313223 & 2 & 1 & & & & 31313223 & 2 & 1 & & & & & & & & & \\
\hline 25 & & & & 31313313 & 1 & 0.5 & 31313313 & 2 & 1 & 31313313 & 3 & 1.5 & 31313313 & 3 & 1.5 & 31313313 & 3 & 1.5 \\
\hline 26 & 31313323 & 5 & 2.5 & 31313323 & 3 & 1.5 & 31313323 & 2 & 1 & & & & & & & & & \\
\hline 27 & 31313333 & 16 & 8.1 & 31313333 & 20 & 10.1 & 31313333 & 94 & 47.5 & 31313333 & 108 & 54.6 & 31313333 & 90 & 45.5 & 31313333 & 109 & 55.1 \\
\hline 28 & 31323233 & 4 & 2 & & & & & & & & & & & & & & & \\
\hline 29 & & & & & & & 31323313 & 1 & 0.5 & & & & & & & & & \\
\hline 30 & & & & & & & 31323333 & 10 & 5.1 & 31323333 & 19 & 9.6 & & & & & & \\
\hline 31 & & & & & & & 31333312 & 1 & 0.5 & & & & & & & & & \\
\hline 32 & 31323313 & 1 & 0.5 & & & & & & & & & & & & & & & \\
\hline 33 & 31323333 & 9 & 4.6 & 31323333 & 14 & 7.1 & & & & & & & & & & & & \\
\hline 34 & 31333223 & 2 & 1 & & & & 31333323 & 1 & 0.5 & & & & & & & & & \\
\hline 35 & 31333323 & 1 & 0.5 & & & & & & & & & & & & & & & \\
\hline 36 & 31333333 & 18 & 9.1 & 31333333 & 19 & 9.6 & 31333333 & 28 & 14.1 & 31333333 & 24 & 12.1 & 31333333 & 37 & 18.7 & 31333333 & 44 & 22.2 \\
\hline 37 & & & & & & & & & & 32113333 & 1 & 0.5 & & & & & & \\
\hline 38 & 32212333 & 3 & 1.5 & & & & & & & & & & & & & & & \\
\hline 39 & & & & & & & & & & & & & & & & 33113333 & 1 & 0.5 \\
\hline 40 & & & & 32213333 & 4 & 2 & & & & & & & & & & & & \\
\hline 41 & 32313333 & 1 & 0.5 & 32313333 & 5 & 2.5 & 32313333 & 1 & 0.5 & 32313333 & 2 & 1 & & & & & & \\
\hline 42 & 32323323 & 1 & 0.5 & 32323323 & 1 & 0.5 & 32323323 & 2 & 1 & 32323323 & 2 & 1 & & & & & & \\
\hline 43 & 32323333 & 1 & 0.5 & 32323333 & 6 & 3.1 & & & & & & & & & & & & \\
\hline 44 & 32333333 & 2 & 1 & 32333333 & 3 & 1.5 & & & & 32333333 & 2 & 1 & & & & & & \\
\hline 45 & & & & 33213333 & 7 & 3.5 & & & & & & & & & & & & \\
\hline 46 & & & & 33313223 & 1 & 0.5 & & & & 33313223 & 1 & 0.5 & & & & & & \\
\hline 47 & & & & 33313323 & 1 & 0.5 & & & & 33313323 & 1 & 0.5 & & & & & & \\
\hline 48 & & & & 33313333 & 9 & 4.6 & & & & 33313333 & 3 & 1.5 & 33313333 & 27 & 13.6 & 33313333 & 7 & 3.5 \\
\hline 49 & & & & 33323313 & 1 & 0.5 & & & & 33323313 & 1 & 0.5 & & & & 33333313 & 1 & 0.5 \\
\hline 50 & & & & 33323323 & 1 & 0.5 & & & & & & & & & & & & \\
\hline 52 & & & & 33323333 & 3 & 1.5 & & & & 33323333 & 2 & 1 & & & & & & \\
\hline 53 & & & & & & & & & & 33333223 & 1 & 0.5 & & & & & & \\
\hline 54 & & & & & & & & & & & & & 33333313 & 1 & 0.5 & & & \\
\hline 55 & & & & 33333323 & 2 & 1 & & & & 33333323 & 3 & 1.5 & & & & & & \\
\hline 56 & & & & 33333333 & 6 & 3 & & & & 33333333 & 2 & 1 & 3333333 & 22 & 11.1 & 33333333 & 12 & 6.1 \\
\hline$\sum \mathrm{N} / \%$ & & 198 & 100 & & 198 & 100 & & 198 & 100 & & 198 & 100 & & 198 & 100 & & 198 & 100 \\
\hline $\begin{array}{l}\text { SPs found } \\
\text { Total SPs possible }\end{array}$ & $\begin{array}{c}29 \\
3^{8}=6561\end{array}$ & & & $\begin{array}{c}28 \\
3^{8}=6561\end{array}$ & & & $\begin{array}{c}19 \\
3^{8}=6561\end{array}$ & & & $\begin{array}{c}23 \\
3^{8}=6561\end{array}$ & & & $\begin{array}{c}10 \\
2^{8}=256\end{array}$ & & & $\begin{array}{c}10 \\
2^{8}=256\end{array}$ & & \\
\hline$\%$ of possible SPs & 0.4 & & & 0.4 & & & 0.3 & & & 0.4 & & & 3.9 & & & 3.9 & & \\
\hline
\end{tabular}




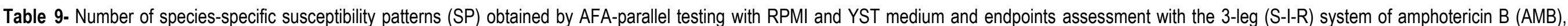

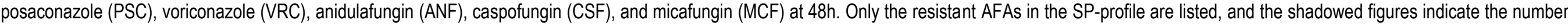
of isolate-populations with the same "resistance pattern" per species. For space reasons S-I mixed patterns are not, respectively, displayed as "S".

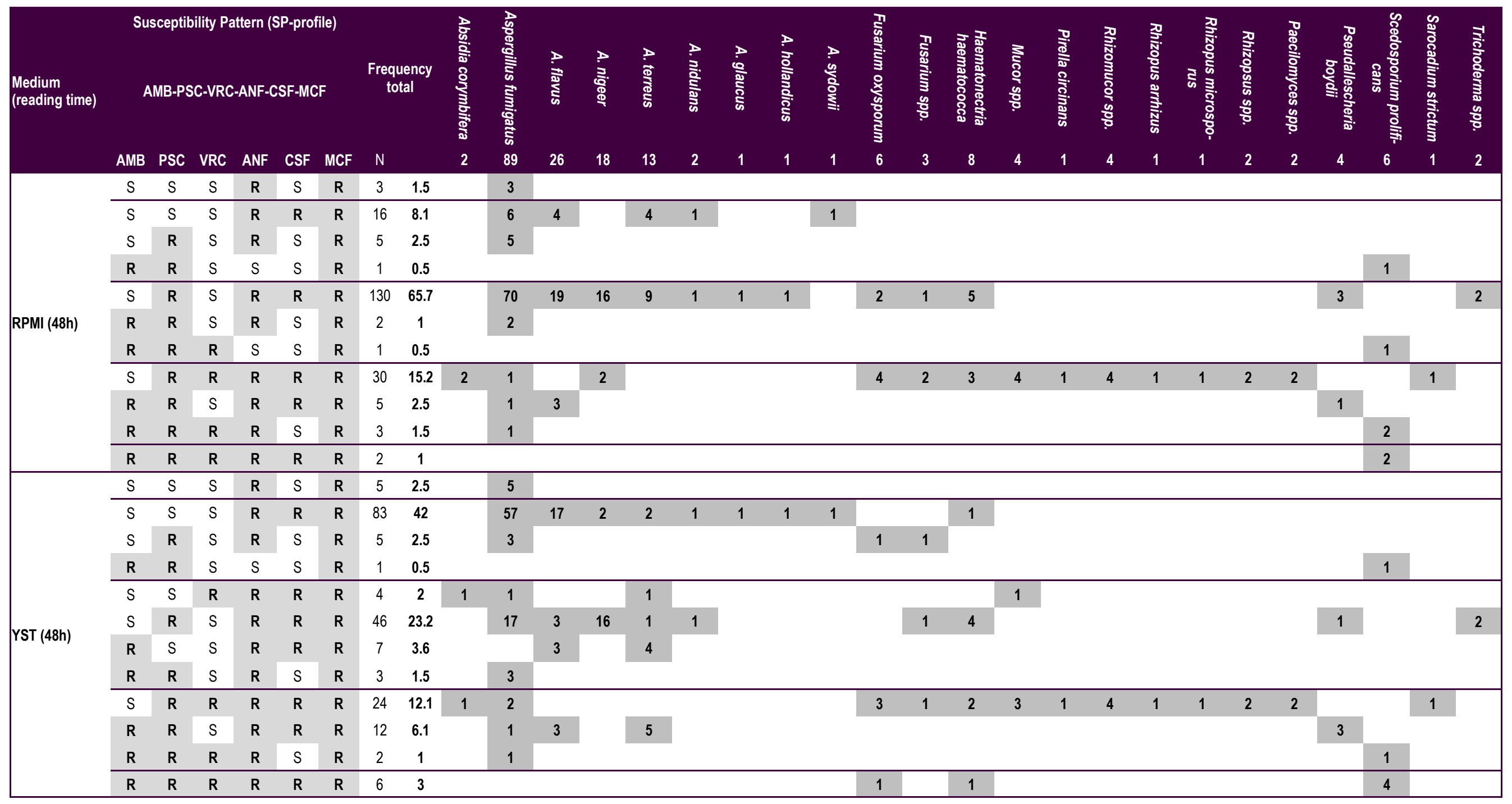

International Journal of Microbiology Research

ISSN: 0975-5276 \& E-ISSN: 0975-9174, Volume 6, Issue 1, 2014 
Table 10- Qualitative and quantitative differences in SP-profiles, which include up to 5 or to 8 resistant antifungal agents (AFAs), are evaluated by SPA. The five to eight-fold multi-resistance (MR) of the AFAs amphotericin B (AMB), flucytosine (FCY), fluconazole (FLC), posaconazole (PSC), voriconazole (VRC), anidulafungin (ANF), caspofungin (CSF), and micafungin (MCF), was evaluated as number and percentage of total strains (\% in round figures) and of number of different species-specific SP-profiles with the as resistant "R" assessed AFAs (shadowed AFA-abbreviations) in the indicated SP-sequence. The shadowed numbers/percentages of the individual SP-profiles indicate the frequency of strains obtained per species with SPA (data from YST medium and the second endpoint reading with 2-leg MIC assessment).

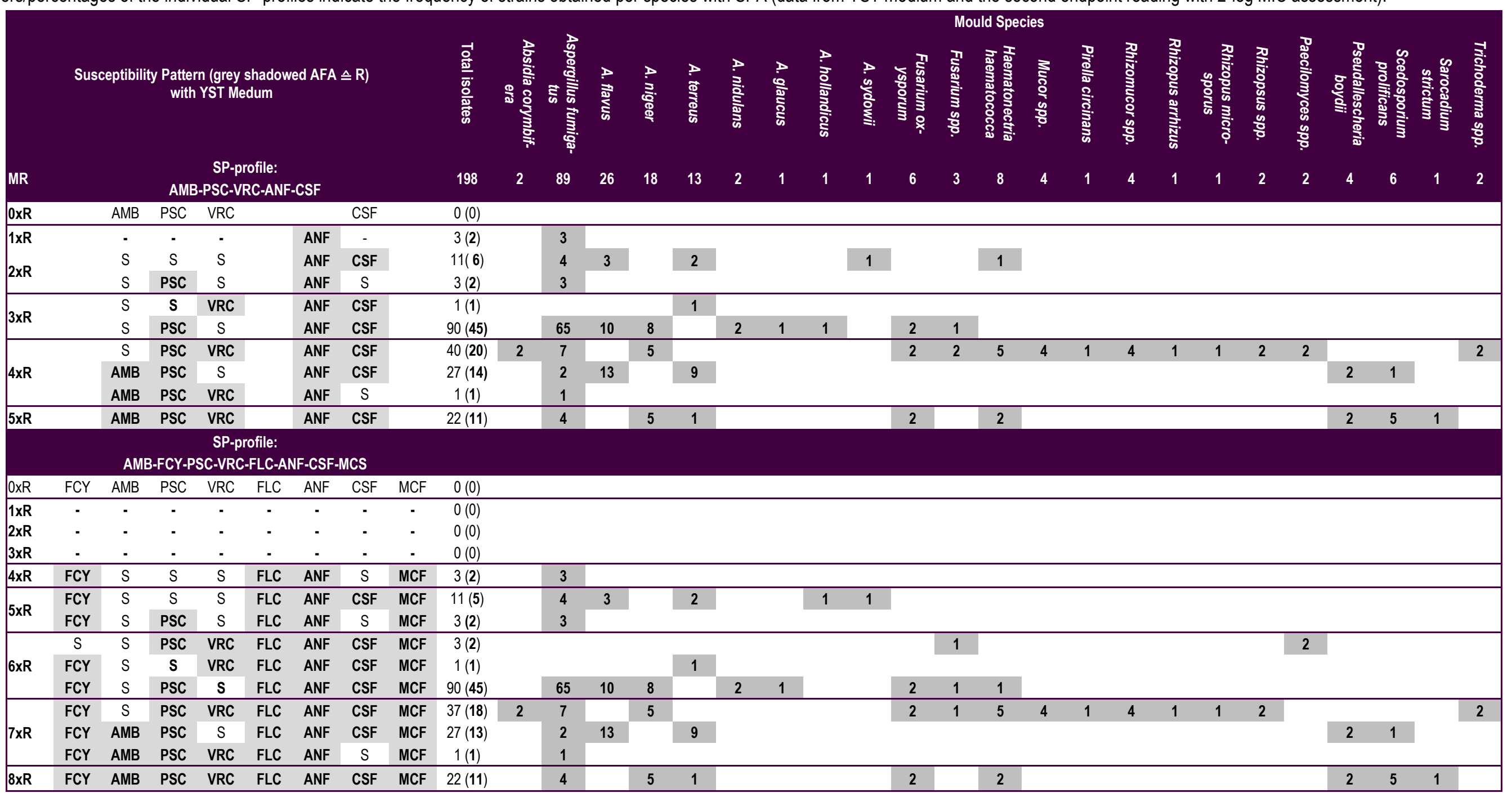

International Journal of Microbiology Research

ISSN: 0975-5276 \& E-ISSN: 0975-9174, Volume 6, Issue 1, 2014 


\section{Parallel and Cross Resistance}

Azole cross-resistance in moulds has been reported [11,20$23,30,34 ; 53-55,78-80]$. SPA demonstrated various multiple resistance-combinations in individual mould isolates, which comprise parallel-resistance among azoles and echinocandins. Individual strain populations demonstrated a twofold to eightfold AFA-crossresistance among the different drug classes. Medium- and endpoint reading-specific differences in the SP-profile were encountered [Table-8]. The medium-dependent SP differences are displayed in [Table-9], when from both media both $48 \mathrm{~h}$ endpointreadings are compared. The number and percentage of all species -specific SP-profiles are shown for the 48h endpoint determinations in [Table-10]. Interestingly, in the collective tested here a complete $(100 \%)$ parallel-resistance between anidulafungin and micafungin and a varied partial parallel-resistance between both, caspofungin and anidulafungin, and caspofungin and micafungin was found [Table-8] to [Table-10]. When the number of SPs obtained with both culture media were compared (3-leg MIC-assessment $\triangleq \mathrm{S}$-I$\mathrm{R})$, after $24 \mathrm{~h}$ incubation 29 SPs were obtained with YST, and 19 SPs with RPMI medium, however, after 48h with YST 28 SPs ($3.5 \%)$, and with RPMI 23 SPs (+14.4\%). When the two-leg assessment system (only R and S) was applied, after $48 \mathrm{~h}$ in both media 10 populations with different SP-profiles were obtained, respectively, after 24h 9 SPs with RPMI, and again 10 SPs with YST medium [Table-8].

An overall trend with RPMI medium to lower MIC-values mirrors the somewhat lower total parallel-resistance rates of echinocandins $(76.7 \% / 73.9 \%$ for YST/RPMI medium), and of azoles (14.4\%/13.9\%). However the parallel-resistance rates within the echinocandins are significant higher $(60 \%)$ when compared to the azoles. This holds also true for the cross-resistance with flucytosine, whereas cross resistance with AMB is about in the same low range as AMB with azoles. Thus, cross-resistance for all isolates was determined to be for AMB and FCY 7.2\%/3.9\% (YST/RPMI), for AMB with azoles $2.8 \% / 1.7 \%$, for AMB with echinocandins $5.0 \% / 2.8 \%$, for FCY with azoles $12.2 \% / 11.7 \%$, for FCY with echinocandins $(73.3 \% / 70.0 \%$, for POS with echinocandins $17.2 \% / 15.6 \%$, for VOR with echinocandins $22.8 \% / 19.4 \%$, for ANI with azoles $14.4 \% / 13.3 \%$, for CAS with azoles $12.8 \% / 11.7 \%$, and for MCA with azoles $14.4 \% / 13.3 \%$ ). Cross-resistance varied significantly among the different species tested, with highest rates in $A$. flavus, A. fumigatus, A. niger, and Glomeromycota (Zygomycetes) [Table-9] and [Table-10].

\section{Discussion}

\section{Inoculum and Endpoint Determination}

For verification of the performance of the new inoculum, MIC testing was performed in parallel with both, YST medium according to DIN and RPMI medium according to EUCAST as described [49]. The new and rapid inoculum preparation was successfully proven for susceptibility testing of the various mould genera and species as reported here, and for dermatophytes (data not shown). The final inoculum of $2.3 \times 10^{4} \mathrm{vu} / \mathrm{ml}$ (mean value of the four labs), which was somewhat higher than the inoculum of $0.4 \times 10^{4}$ to $5 \times 10^{4} \mathrm{cfu} / \mathrm{ml}$ as proposed by CLSI M38-A2 [65], and lower than the $2 \times 10^{5}$ to $5 \times 10^{5} \mathrm{cfu} / \mathrm{ml}$ as recommended by EUCAST EDef9 [59], was sufficient to perform reproducible and comparable MICs for all the tested moulds in both media. Therefore, by direct use of freshly grown mould cultures (pre) testing times for filamentous fungi were significantly reduced and all moulds could be tested within $72 \mathrm{~h}$ in the microdilution system. MIC endpoints were stable and reproducible to be read in both media tested. Therefore this inoculum preparation method may be successfully extended to other sporogenous and a-sporogenous hyphae-forming fungi. This may be supported by the results of Manavathu, et al [62-63], demonstrating that either germinated or ungerminated conidia could be used as inocula for in vitro susceptibility studies because both revealed identical results from Aspergillus fumigatus. In addition, Anatachopoulos, et al [64] and Bowman, et al [82] showed that caspofungin kills growing cells, and that differentially increased concentrations of echinocandins are necessary to affect germinated versus nongerminated conidia. Even with a10-fold amount of drug over the MIC, outgrow of conidia and hyphae had been determined [67]. Despite that there should be no differences in using geminated and ungerminated spores in susceptibility testing [62], there is a wide range of 2 dilutions for the concordance of their MFCs and MICs as reported by Espinel-Ingroff [83]. Additionally, the "more than $99 \%$ inhibition of all isolates by $0.063 \mathrm{mg} / \mathrm{L}$ " for all three echinocandins [72] differs from the $0 \%$ to $3 \%$ (MCF, ANF) of strains found with a MIC of $0.063 \mathrm{mg} / \mathrm{l}$ [Table-6] and [Table-7c]. The reported $88 \%$ to $94 \%$ inhibition of the azoles PSC and VRC by MICs of $\leq 1 \mathrm{mg} / \mathrm{L}$ in other studies [39,40,53], are somewhat higher than those with $70 \%$ to $80 \%$ (48h values) in [Table-6] and [Table-7b]. These deviations may be due to the above mentioned facts, the use of the conidia dependent (CLSI/EUCAST) methodology, and the reporting of susceptibility as MECs.

\section{Susceptibility Testing - MICs Distributions}

Although external studies reported to adhere to the CLSI or EUCAST standard, respectively, almost all authors either reclaimed some deviations or tested differing MIC ranges, and enrolled strain collectives. Despite these differences, it is demonstrated by [Table7a] and [Table-7b] that at least the characteristic MICs of amphotericin $B$ and-the azoles match quite well for the different moulds and different international studies. On the other hand, MICs for azoles and AMB of this study are found to be more comparable, respectively quite similar, e.g. to those presented by Sabatelli, et al [41] and other international studies [Table-7a] and [Table-7b]. This is in agreement with the fact that YST and RPMI 1640 media showed very close MICs, and more than $92 \%$ of the isolates were within $\pm 1 \log _{2}$-dilution. As reported elsewhere, the $48 \mathrm{~h}$ MICs were slightly higher compared to $24 \mathrm{~h}$ MICs. As shown by RodriguezTudela, et al for the EUCAST method [35] that resistance to Aspergillus may be detected only after $48 \mathrm{~h}$ incubation, the data here indicate at least for fast-growing hyphomycetes and the "new inoculum" method that most elevated mould MICs may be detected already within $48 \mathrm{~h}$.

\section{Minimum-Effective Concentration}

MEC determinations should replace the MIC to describe an antifungal susceptibility endpoint for echinocandins. Although hyphae are in general the infectious forms of filamentous fungi, their spores (conidia) are used in susceptibility testings recommended by CLSI and EUCAST, which may require for some species the induction of sporulation. However, for the determination of inhibition (MICs) the detection of outgrown conidia, and the assessment of "stubby and highly branched", respectively distorted hyphae $[84,85,86]$ is necessary. The complex mechanism and the differences in germination of different Aspergillus species [87], as well as differences in the surface structure and of proteins in dormant 
and swelling (pre-swelled) conidia [88] may significantly bias in vitro susceptibility testing results. As documented for dermatophytes, that the MIC of hyphae and conidia with their thicker cell walls differ significantly, as do the macro- (MAC) and micro-conidia (MRC) [89]. Therefore also for spore-forming moulds the separation of the larger MAC and hyphae from the smaller MRC has to be performed [86]. However as reported, the method recommended by CLSI is insufficient to achieve reliable results for the more reproducible MRC-tests [90]. Due to these differences, and lack of data, the photometric adjustment of the target transmission to the conidial size for less common moulds has to be determined by "trial and error" [86]. It has to be mentioned too, that, e.g., Aspergillus terreus produces accessory conidia (aleurioconidia), both in vitro and in vivo [91]. They are distinct from phialidic conidia in colour and cell size, and demonstrate differential rates of germination and hydrophobicity [92]. Additionally, their susceptibility status to antifungal agents is yet unknown. Furthermore, for triazoles a varying ability to inhibit conidia formation in Aspergillus species has also been published [93], and the Etest endpoint for Aspergillus isolates is reported to prove poor conformity with the "reference minimum effective concentration" [94]. In addition, due to the possible technician-dependent bias in MEC determinations from different laboratories, the resulting MEC value may be rather subjective when "inhibition of growth is not necessarily complete" [67]. Therefore the correlation to MICs, and as mentioned by Arendrup, et al [95]: the "correlation of wild type MECs to in vivo susceptibilities" has to be questioned. Thus, MIC differences in the literature, and as displayed in [Table-6] to [Table-7c] may not only be methodology- and conidia-dependent but also due to reporting (biased) results as MECs, or as "high-minimum-effective-concentrations (high-MECs), as visual determined growth endpoints, as $50 \%$ growth inhibition by photometric means, or contrastingly commonly as $80 \%-100 \%$ visual growth inhibition. Although a fairly high agreement for $A M B$ testing with the CLSI and a colorimetric microdilution method was observed, instead of an expected higher accuracy and reproducibility for the spectrophotometric endpoint method, a lack of sensitivity was detected [96]. These discrepancies raise the question whether all these differently reported endpoint determinations may reflect a standardized, unique, and comparable "minimum inhibitory concentration", and whether endpoint assessments compare to "susceptible" or "resistant".

Further obstacles in comparison of resistance-results may be encountered due to the quite differing fungal collectives tested, and the taxonomy used. Because to the "one fungus one name" principle, several new taxons may appear, respectively old ones may change their name, which will result in altered antimicrobial AFA effectiveness and MIC-ranges. As shown, that phylogenetic relationships matter [57], which is also valid for moulds [4] the currently valid nomenclature $[93,94]$ was used in this paper [Table-1], which may add to further divergences here and in the future. In this context it has to be reminded that even when it has been demonstrated that e.g. the in vitro results of CLSI and EUCAST determinations are in large part $(>90 \%)$ within $\pm 1 \log _{2}$-dilution, as also shown here for the MIC results with the DIN and EUCAST medium, that this widely accepted range of variation of twofold dilutions in microdilution tests indicates a range differing by $300 \%$ [99].

\section{Epidemiological Cut-off Values}

ECVs have been defined for some Aspergillus species for triazoles, caspofungin, and amphotericin $B$ in the absence of clinical breakpoints (CBPs). These ECVs should indicate strains with resistance mutations and reduced antifungal activity (non-wild type), and distinguish them from the "wild type" (WT) populations (without acquired mutations), however are used increasingly instead of CBPs for MIC assessments. The proof, that WT-strains do not harbour pre-step mutations to resistance or are without (down regulated) resistance genes cannot be found in the literature. Although the definitions of WT and ECVs have been defined previously [100-103], they appear in the literature as various terms and a mix up of different versions (e.g., "wild type cutoff limits", "wildtype cut-off values", "wild-type upper limits", "wild-type lower and upper cut-off values", "wild-type minimum effective concentration", "epidemiological cut-off limits", "epidemiological cut off values" (ECOFF), "epidemiological upper and lower cut-off values"). Often, they are also used in the context with "epidemiological", "microbiological", and "clinical breakpoints", partly without any differentiation or appropriate reference. Even more confusion is created by the fact that ECVs/ECOFFs from CLSI and EUCAST are based on different MIC "mean-values", which can also lead to different or even incomparable results. This is particularly relevant when larger collectives have to be evaluated (data not shown). A tabled comparison by Arikan-Akdaki [104] of ECVs/ECOFFS issued by CLSI and EUCAST demonstrate differing values. Even when MCVs were calculated from the modal MICs in a comparative study, each obtained from both methods performed in parallel, different ECVs were encountered [105]. As examples, CLSI ECVs are based on modal MICs $[106,107]$, whereas those by EUCAST have been described to be also based on modal [78], however, mainly on median MIC-values $\left(\mathrm{MIC}_{50}\right)[108,109]$. Additionally, epidemiological cut-off values, rather than preliminary breakpoints, are recommended until the necessary information on CBPs, respectively correlation between microbiological and clinical outcome becomes available [110] (up today, no clinical study published has been performed where this criteria was explicitly included in the study protocol). Another proposal in this regard is to distinguish between phenotype- (pan-azole and multi-azole resistant) and genotype resistance (gene-related resistance with a corresponding phenotype resistance to an individual AFA) [52]. Based on these facts as well on the absence of an international, validated routine susceptibility test method for fungi and the still missing unique CBPs, the echinocandin (and all other commonly used AFA) susceptibility data have to be dealt and compared with great care [Table-6], [Table-7a] to [Table-7c]. Although the usefulness of ECVs in resistance surveillance, epidemiological programs, and as an important step in the establishment of clinical breakpoints [100] is not questioned, they shall not replace the clinical breakpoints. Moreover, as requested by Simjee, et al $[111,112]$ all these "terms in regard to "resistance shall not be mixed up in assessment and reporting of MIC susceptibility/resistance data".

\section{Parallel and Cross Resistance}

Parallel-resistance, commonly in the literature referred to as crossresistance, have been reported for triazoles and echinocandins. However, such data were not analysed quantitatively but rather deduced from percentaged MIC-distributions or molecular diagnostic probing $[53,116,117]$. Examples of, azole-cross-resistance [53,78,118-122], of isolates with reduced susceptibility/resistance to echinocandins $[19,20,25,32,33,53,81,116,120$, and the occurrence of amphotericin B-resistant strains $[9,16,31,38,40-43,63,71$. 72] have been published. That there is a solid level of parallel- and/ 
or cross-resistance among the systemically used drugs could be shown here [Table-8] \& [Table-10]. As the possible cross-linkages might be quite high (theoretically for the three assessment categories S-I-R, and the 8 AFAs there are $3^{8}=6561$ combinations possible). That only a few of the possible SPs are found in nature is clearly seen in [Table-8]. To demonstrate for comparison purposes exemplarily the outcome of susceptibility pattern analysis, for all 8 AFAs, and with a set of five drugs where the AFAs with no or little action to moulds in this study were excluded, the SP-profiles were displayed in [Table-10]. Here, and in addition to reduce the theoretical amount of possible cross-linkages, only the "S" and "R" category with the breakpoints $S: \leq 1$, and $R:>1$, were applied (reduced possible cross-resistances: $2^{5}=32$ in contrast to $2^{8}=256$ ).

That, cross-resistance profiles provide a strategy to predict resistance mechanisms have been demonstrated by Hill, et al [122]. Variation in the patterns of cross-resistance to the distinct drug combinations, and differences in the level of resistance to the drug combinations within and between different species was observed [Table-9] and [Table-10]. The difference, when only a part of the in parallel tested drugs ( 5 items) is compared to the complete set (8 AFA) is seen in [Table-10]. Whereas in the 5-AFA set populations with single, double or triple AFA-resistances appear, they are absent in the complete 8-drug set because they are masked by the additional parallel and/or cross-resistant AFAs in the SP-profile. Thus it is quite obvious that when additional drugs to which the isolates show elevated MICs are included in the test panel, more populations with varying SP's, respectively altered specific crossresistance profiles (SPs) are obtained, which resemble the variation in levels of resistance to the individual drugs, the isolates were initially treated. This is amplified by a prolonged incubation time [Table 8], and may be a reason of the reported low efficacy, e.g. of combinations of azoles and echinocandins [123,124]. Additionally, without, during or after azole treatment there is an increase in the evolution of azole cross-resistance (mechanisms) with concomitant treatment failure reported by Howard, et al [24] and Bueid, et al [125]. That more strains with higher MICs for PSC and VRC were encountered [Table-6] and [Table-7] may be due to a higher amount of isolates with cyp $15 \mathrm{~A}$ gene based resistance mechanisms which have been spread in Europe [35,52,119,122-132], and also been laboratory-confirmed in Germany $[79,80]$. The same may apply for the echinocandins, of which some isolates have quite lower susceptibility rates compared to other studies [Table-6] and [Table-7c], when strains with FKS1 gene based resistances $[95,120,133,134]$ may be present. As at the time when the study was performed the tools to detect these mutations were not available, therefore these investigations have not been carried out.

The multitude and variations of distinct drug combinations in species-specific SPs, and the tendency of higher MIC values in the SPs to be found with increasing incubation times, together with the variations within a SP-category may indicate that more than one resistance mechanism is responsible for high MICs-levels, respectively overall resistance. In these populations, described resistance genes or mechanisms are harboured, induced, and/or up-regulated and expressed, indicating that changes in populations between in $24 \mathrm{~h}$ versus $48 \mathrm{~h}$ endpoint reading (because the actual incubation times are longer) may not be only artefacts [78]. This is supported by the fact that the fungal population size is most dependent on changes in the mycelial growth rate $[135,136]$, and may be underpinned by the determination of wild-type MICs according to other authors $[78,84,109,118]$. It also has been shown that the degree of inhibition is important for adaptive (resistance) mutations [137,138], which may lead to resistance or at least as shown in Saccharomyces cerevisiae, to minimize the impact of azoles to their target [139], as those single-gene mutations are sufficient to create high levels of resistance [140,142]. That azole pre-exposure affects Aspergillus fumigatus populations in patients has been demonstrated too [143-145].

Interestingly, the "wild-type (WT) cut-off values" (according to Kahlmeter [100] identical to "epidemiological cut off values") published $[78,84,105,118,146,147]$, parallel the MICs fifth percentile $\left(\mathrm{MIC}_{5}\right)$ in which the WT-populations maybe found, and the MICs 75th percentile $\left(M C_{75}\right)$ which equals in most cases the ECV or ECOFF [Table-4] \& [Table-5]; [Fig-1], [Fig-2] \& Fig-3]. Whereas the percentage of susceptible/resistant isolates changes by altering the chosen breakpoints, the population based parameters (e.g., $\mathrm{MIC}_{5}$, $\mathrm{MIC}_{75}$ ) will not change, which is supported by the data presented here, i.e., whilst the populations with characteristic of susceptibility patterns will not change significantly with the application of lower breakpoints, the percentage of resistance together with the percentages of parallel- and cross-resistance will increase [Table-8] and [Table-10]. Furthermore, the increasing reports of acquired resistance in $A$. fumigatus of environmental origin have to be considered for an ongoing increase in cross-resistance $[37,127,128$, 148], and that triazoles used as fungicides can induce azole crossresistance in A. fumigatus [149].

\section{Conclusions}

The data demonstrate that this novel inoculum preparation method for susceptibility testing of hyphae-forming fungi using prefabricated microdilution plates showed in essence comparable MICs to those obtained by EUCAST and CLSI with the conidia method. However, in the light of the emerging issue of invasive mycoses, the time-saving effect of this new approach may contribute substantially to an improved impact of susceptibility testing and antifungal resistance screening of Aspergillus and other moulds. The media recommended by DIN (YST) and EUCAST (RPMI 1640 with $2 \%$ glucose) for susceptibility testing of fungi performed similarly, with an essential agreement of $>92 \%$ for all 13 genera and 24 species. However there is still a need for media improvement for faster and more unique growth rates and more reliable endpoint determinations. It could be shown by susceptibility pattern analysis that with different culture media, varying inocula, and longer incubation times not only a tendency to higher MIC-values has to be encountered but also a qualitatively and quantitatively change in isolate populations with altered susceptibility patterns could be determined. The fact that correct identification and use of the current taxonomy is essential in order to receive comparable MIC results may clearly be seen from the data presented. Therefore, together with the adherence to the current valid nomenclature, a validated international (ISO) routine method with both, clear-cut defined and appropriate breakpoints for microbiological (epidemiological) and clinical breakpoints would most urgently be needed, at least for all used systemic antifungal agents in order to compare and confidentially make use of the results published locally and internationally. In addition, the use of a suitable susceptibility patterns analysis indicating distinct cross-resistance profiles and their population-specific levels may help to detect and understand evolving resistance mechanisms and help in the decision to select appropriate or complementary drugs for therapy. 


\section{List of Abbreviations}

AFA(s): antifungal agent(s)

AMA(s): antimicrobial agent(s)

AMB: amphotericin B

ANF: anidulafungin

ATCC: American Type Culture Collection

cfu: colony forming units

CR: cross-resistance

CSF: caspofungin

CLSI: Clinical Laboratory Standards Institute

DIN: Deutsches Institut für Normung e.V.

EA: essential agreement

ECOFF: epidemiological cut-off value

ECV: epidemiological cut-off value

EUCAST: European national breakpoint committees

FCY: flucytosine

FLC: fluconazole

IRQ: interquartile range

MCF: micafungin

MAC: macro-conidia

MEC: minimum effective concentration

MIC: minimum inhibitory concentration

MRC: micro-conidia

MR: multi (multiple) resistance

n.a.: not available

n.t.: not tested

PR: parallel-resistance

PSC: posaconazole

RPMI: Roswell Park Memorial Institute Medium

SAB: Sabouraud agar plate

S-I-R: susceptible (S), intermediate (I), resistant ${ }^{\circledR}$

SP: susceptibility pattern

SPA: susceptibility pattern analysis

VRC: voriconazole

vu: viable unit

YST: Yeast Sensitivity Test medium

\section{Acknowledgments}

Free of charge were provided the antifungal agents anidulafungin, fluconazole, and voriconazole by Pfizer $\mathrm{GmbH}$ (Berlin, Germany), caspofungin by MSD Sharp \& Dohme GmbH (Munich, Germany), posaconazole by Essex Pharma $\mathrm{GmbH}$ (Munich, Germany), and micafungin by Astellas Pharma GmbH (Munich, Germany).

\section{Competing Interests}

The authors declare that no writing assistance was utilized, and they have no conflict of interests in preparing and publishing this manuscript.

\section{Funding}

To perform and report this study, no financial support from pharmaceutical or other companies or any non-commercial, national, or international funding source was received.

\section{References}

[1] Richardson M.D. (2005) Journal of Antimicrobial Chemotherapy, 56 (1), i5-i11.

[2] Nayak N. (2008) Nepal Medical College Journal, 10(1), 48-63.

[3] Revankar S.G. (2014) Infectious Diseases\& Antimicrobial Agents, $\mathrm{t} 15$.

[4] Quan C., Spellber B. (2010) Proceedings of the American Thoracic Society, 7, 210-215.

[5] Kwon-Chung K.J. (2012) Clinical Infectious Diseases, 54(1), S8-S15

[6] Pfaller M.A., Diekema D.J. (2004) Journal of Clinical Microbiology, 42, 4419-4431.

[7] Walsh T.J., Groll A. \& Hiemenz J. (2004) Clinical Microbiology and Infection, 10(1), 48-66.

[8] Lass-Flörl C. (2009) Mycoses, 52, 197-205.

[9] Binder U. \& Lass-Flörl C. (2011) Mediterranean Journal of Haematology and Infectious Diseases, 3, e20110016.

[10]Koo S., Klompas M. \& Marty F.M. (2010) Medical Mycology, 48, 769-774.

[11]Revankar S.G. (2012) Infectious Disease \& Antimicrobial Agents, f05.

[12]Saiyarin J. \& Chayakulkeeree M.D. (2013) Journal of Infectious Diseases and Antimicrobial Agents, 30, 109-125.

[13]Schieffelin J.S., Garcia-Diaz J.B., Loss Jr. G.E., Beckman E.N., Keller R.A., Staffeld-Coit C., Garces J.C. \& Pankey G.A. (2014) Transplant Infectious Disease, 16, 270-278.

[14]Gubbins P.O. \& Anaissie E.J. (2009) Antifungal Therapy, Clinical Mycology 2nd ed., 167-199, Churchill-Livingstone, New York, NY.

[15]Fera M.T., La Camera E., De Sarro A. (2009) Expert Review of Anti-infective Therapy, 7, 981-989.

[16]Pappas P.G., Alexander B.D., Andes D.R., Hadley S., Kauffman C.A., Freifeld A., Anaissie E.J., Brumble L.M., Herwaldt L., Ito J., Kontoyiannis D., Lyon G.M., Marr K.A., Morrison V.A., Park B.J., Patterson T.F., Perl T.M., Oster R.A., Schuster M.G., Walker R., Walsh T.J., Wannemuehler K.A. \& Chillera T.M. (2010) Clinical Infectious Diseases, 50, 1101-1111.

[17]Wingard J.R. (2005) Current Opinion in Oncology, 17, 89-92.

[18]Alcazar-Fuoli L., Rodríguez-Tudela J.L. \& Mellado E. (2010) Current Fungal Infection Reports, 2(1), 36-42.

[19]Smith J.A. \& Kauffman C.A. (2012) Respirology, 17, 913-926.

[20]Warris A., Weemaes C. \& Verweij P.E. (2002) New England Journal of Medicine, 347, 2173-2174.

[21]Verweij P.E., Mellado E. \& Melchers W.J.G. (2007) New England Journal of Medicine, 356, 1481-1483.

[22]Denning D.W., Venkateswarlu K., Oakley K.L., Anderson M.J., Manning N.J., Stevens D.A., Warnock D.W. and Kelly S.L. (1997) Antimicrobial Agents and Chemotherapy, 41(6), 13641368.

[23]Nascimento A.M., Goldman G.H., Park S., Marras S.A.E., Delmas G., Oza U., Lolans K., Dudley M.N., Mann P.A. \& Perlin D.S. (2003) Antimicrobial Agents and Chemotherapy, 47, 1719 -1726 .

[24]Howard S.J., Webster I., Moore C.B., Gardiner R.E., Park S., 
Perlin D.S. \& Denning D.W. (2006) International Journal of Antimicrobial Agents, 28(5), 450-453.

[25]Lass-Flörl C. \& Perkhofer S. (2008) Mycoses, 51, 437-446.

[26]Kanafani Z.A. \& Perfect J.R. (2008) Antimicrobial Agents and Chemotherapy, 54, 1864-1871.

[27]Lass-Flörl C. (2010) Future Microbiology, 5, 789-799.

[28]Howard S., Cerar D., Anderson M., Albarrag A., Fisher M., Pasqualotto A., Laverdiere M., Arendrup M.C., Perlin D. \& Denning D. (2009) Emerging Infectious Diseases, 15, 10681076.

[29]Howard S.J. \& Arendrup M.C. (2010) Medical Mycology, 49(1), S90-S95.

[30]Arendrup M.C., Mavridou E., Mortensen K., Snelders E., Frimodt-Møller N., Khan H., Melchers W.J.G. \& Verweij P.E. (2010) PLoS ONE, 5(4), e10080.

[31]Lass-Flörl C. (2010) Current Fungal Infection Reports, 3(4), 236-242.

[32]Manavathu E.K., Cutright J.L. \& Chandrasekar P.H. (2005) Antimicrobial Agents and Chemotherapy, 49, 428-430.

[33]van Leer-Buter C., Takes R.P., Hebeda K.M., Melchers W.J.G. \& Verweij P.E. (2007) Lancet, 370(9581), 102.

[34]van der Linden J.W.M., Jansen R.R., Bresters D., Visser C.E., Geerlings S.E., Kuijper E.J., Melchers W.J.G. \& Verweij P.E. (2009) Clinical Infectious Diseases, 48, 1111-1113.

[35]Vermeulen E., Lagrou K. \& Verweij P.E. (2013) Current Opinion in Infectious Diseases, 26(6), 493-500.

[36]Lelièvre L., Groh M., Angebault C., Maherault A.C., Didier E. \& Bougnoux M.E. (2013) Médecine et Maladies Infectieuses, 43, 139-245.

[37]Chowdhary A., Kathuria S., Xu J. \& Meis J.F. (2013) PLoS Pathogens, 9(11).

[38]Kontoyiannis D.P., Lewis R.E., May G.S., Osherov N. \& Rinaldi M.G. (2002) Mycoses, 45(9-10), 406-407.

[39]Walsh T.J., Petraitis V., Petraitiene R., Field-Ridley A., Sutton D., Ghannoum M., Sein T., Schaufele R., J., Bacher J., Casler H., Armstrong D., Espinel-Ingroff A., Rinaldi M.G. \& Lyman C.A. (2003) Journal of Infectious Diseases, 188(2), 305-319.

[40]Tortorano A.M., Prigitano A., Dho G., Biraghi E., Stevens D.A,. Ghannoum M., Nolard N. \& Viviani M.A. (2008) Journal of Chemotherapy, 20(6), 756-757.

[41]Hof H. \& Dietz A. (2009) International Journal of Antimicrobial Agents, 33(3), 285-286.

[42]Blum G., Hörtnagl C., Jukic E., Erbeznik T., Pümpel T., Dietrich H., Nagl M., Speth C., Rambach G. \& Lass-Flörl C. (2008) Antimicrobial Agents and Chemotherapy, 52(4), 1553-1555.

[43]Blum G., Perkhofer S., Haas H., Schrettl M., Würzner R., Dierich M.P. \& Lass-Flörl C. (2013) Chemotherapy, 57, 1583-1588.

[44]Schmalreck A.F., Kottmann I., Reiser A., Ruffer U., Scharr E. \& Vanca E. (1995) Mycoses, 38, 359-368.

[45]Espinel-Ingroff A., Canton E. \& Pemán J. (2009) Current Fungal Infection Reports, 3, 133-141.

[46]Hall G. (2012) Interactions of Yeasts, Moulds, and Antifungal Agents - How to detect Resistance, Springer New York Dordrecht Heidelberg London.
[47]Balajee A.S. \& Marr K.A. (2002) Journal of Clinical Microbiology, 40, 2741-2745.

[48]Cuenca-Estrella M. \& Rodriguez-Tudela J.L. (2010) Expert Review of Anti-infective Therapy, 8, 267-276.

[49]Schmalreck A., Willinger B., Czaika V., Fegeler W., Becker K., Blum G. \& Lass-Flörl C. (2012) Mycopatholgia, 174(5-6), 467474.

[50]DIN 58940-81 (2002) Medizinische Mikrobiologie - Empfindlichkeitsprüfung von mikrobiellen Krankheitserregern gegen Chemotherapeutika. Teil 84: Mikrodilution - Spezielle Anforderungen an die Testung von Pilzen gegen Antimykotika. Normenausschuss Medizin (NAMed) im DIN Deutsches Institut für Normung e.V., Berlin.

[51]Clinical and Laboratory Standards Institute (2002) Reference method for broth dilution antifungal susceptibility testing of filamentous fungi, Approved standard, Document M38-A, Wayne, PA.

[52]Verweij P.E., Howard S.J., Melchers J.G. \& Denning D.W. (2009) Drug Resistance Updates, 12(6), 141-147.

[53]Pfaller M.A., Messer S.A., Boyken L., Rice C., Tendolkar S., Hollis R.J. \& Diekema D.J. (2008) Journal of Clinical Microbiology, 46, 2568-2572.

[54]Vandeputte P., Ferrari S. \& Coste A.T. (2012) International Journal of Microbiology, 713687.

[55]Hill J.A., Ammar R., Torti D., Nislow C. \& Cowen L.E. (2013) PLoS Genetics, 9(4), e1003390.

[56]Schmalreck A.F., Willinger B., Haase G., Blum G., Lass-Flörl C., Fegeler W., Becker K. \& Antifungal Susceptibility Testing (AFST) Study Group (2012) Mycoses, 55, e124-e137.

[57]Schmalreck A.F., Lackner M., Becker K., Fegeler W., Czaika V., Ulmer H. \& Lass-Flörl C. (2013) Antimicrobial Agents and Chemotherapy, 58(3), 1575-1585.

[58]Subcommittee on Antifungal Susceptibility Testing (AFST) of the ESCMID European Committee for Antimicrobial Susceptibility Testing (EUCAST) (2008) Clinical Microbiology and Infection, 14, 398-405.

[59]Subcommittee on Antifungal Susceptibility Testing (AFST) of the ESCMID European Committee for Antimicrobial Susceptibility Testing (EUCAST) (2008) Clinical Microbiology and Infection, 14, 982-984.

[60]Arendrup M.C., Garcia-Effron G., Lass-Flörl C., Gomez-Lopez A., Rodriguez-Tudela J.L., Cuenca-Estrella M. \& Perlin D.S. (2009) Antimicrobial Agents and Chemotherapy, 54, 426-439.

[61]Fegeler W., Lintz D., Ritzerfeld W. (1988) Zentralblatt für Bakteriologie und Hygiene A, 270, 153-159.

[62]Manavathu E.K., Cutright J., Chandrasekar P.H. (1999) Journal of Clinical Microbiology, 37, 858-861.

[63]Manavathu E.K., Cutright J.L., Loebenberg D. \& Chandrasekar P.H. (2000) Journal of Antimicrobial Chemotherapy, 46, 229234.

[64]Antachopoulos C., Meletiadis J., Sein T., Roilides E., Walsh T.J. (2008) Antimicrobial Agents and Chemotherapy, 52, 321328.

[65]Clinical and Laboratory Standards Institute (2008) Reference method for broth dilution antifungal susceptibility testing for 
filamentous fungi; approved standard. Document M38-A2, Wayne, PA.

[66]Kurtz M.B., Heath I.B., Marrinan J., Dreikorn S., Onishi J. \& Douglas C. (1994) Antimicrobial Agents and Chemotherapy, $38,1480-1489$.

[67]Ingham C.J. \& Schneeberger P.M. (2012) PLOS ONE, 7(4), e35478.

[68]Lass-Flörl C., Mayr A., Perkhofer S., Hinterberger G., Hausdorfer J., Speth C. \& Fille M. (2008) Antimicrobial Agents Chemotherapy, 52(10), 3637-3641.

[69]Cuenca-Estrella M., Gomez-Lopez A., Mellado E., Buitrago M., Monzon A. \& Rodriguez Tudela J.L. (2006) Antimicrobial Agents Chemotherapy, 50(3), 917-921.

[70]Alastruey-Izquierdo A., Mellado E., Peláz T., Pemá J., Zapico S., Alvarez M., Rodriguez-Tuedla J.L., Cuenca-Estrella M. \& FILOP Study Group (2013) Antimicrobial Agents and Chemotherapy, 57(7), 3380-3387.

[71]Sabatelli F., Patel R., Mann P.A., Mendrick C.A., Norris C.C., Hare R., Loeberg D., Black T.A. \& McNicholas P.M. (2006) Antimicrobial Agents and Chemotherapy, 50(6), 2009-2015.

[72]Diekema D.J. Messer S.A. Hollis R.J., Jones R.N. \& Pfaller M.A. (2003) Journal of Clinical Microbiology, 41(8), 3623-3626.

[73]Cuenca-Estrella M., Gomez-Lopez A., Mellado E., GarciaEffron G., Momnzon., Rodriguez Tuedla J.L. (2005) Antimicrobial Agents and Chemotherapy, 49(12), 5136-5138.

[74]Cuenca-Estrella M., Gomez-Lopez A., Mellado E., Monzon A., Buitrago M.J. \& Rodriguez-Tuedla J.L. (2009) Antimicrobial Agents and Chemotherapy, 53(5), 2192-2195.

[75]Shi J.Y., Xu Y.C., Shi Y., Lü H.X., Liu Y., Zhao W.S., Chen D.M., Xi L.Y., Zhou X., Wang H. \& Guo L.N. (2010) Chinese Medical Journal, 123(19), 2706-2709.

[76]Dannaoui E., Meletiatis J., Mouton J.W., Meis F.G.M., Verweij P.E. \& the Eurofung Network (2003) Journal of Antimicrobial Chemotherapy, 51, 45-52.

[77]Lockhart S.R., Zimbeck A.J., Baddley J.W., Marr K.A, Andes D.R., Walsh T.J., Kauffman C.A., Kontoyiannis D.P., Ito J.I., Pappas P.G. \& Chiller T. (2011) Antimicrobial Agents Chemotherapy, 55(8), 3944-3946.

[78]Rodriguez-Tudela J.L., Alcazar-Fuoli L., Mellado E., AlastrueyIzquierdo A., Monzon A. \& Cunca-Estrella M. (2008) Antimicrobial Agents and Chemotherapy, 52, 2468-2472.

[79]Walker L.A., Gow N.A.R., Munro C.A. (2010) Fungal Genetics and Biology, 47, 117-126.

[80]Rath P.M., Buchheidt D., Spiess B., Arfanis E., Buer J. \& Steinmann J. (2012) Antimicrobial Agents and Chemotherapy, 56, 6060-6061.

[81]Bader 0., Weig W., Reichard U., Lugert R., Kuhns M., Christner M., Held J., Peter S., Schumacher U., Buchheidt D., Tintelnot K., Groß U. \& Mykol.LabNet-D Partners (2012) Antimicrobial Agents and Chemotherapy, 67(8), 3513-3517.

[82]Bowman J.C., Hicks S., Kurtz M.B., Rosen H., Schmatz D.M., Liberator P.A. \& Douglas C.M. (2002) Antimicrobial Agents and Chemotherapy, 46, 3001-3012.

[83]Espinel-Ingroff A. (2001) Antimicrobial Agents and Chemotherapy, 45(2), 605-607.
[84]Pfaller M.A., Boyken L., Hollis R.J., Kroeger J., Messer S.A., Tendolkar S. \& Diekema D.J. (2010) Diagnostic Microbiology and Infectious Diseases, 67, 55-60.

[85]Odds F.C., Motyl M., Andrade R.H., Bille J., Cantón E., Cuenca -Estrella M., Davidson A., Durussel C., Ellis D., Foraker E., Fothergill A.W., Ghannoum M.A., Giacobbe R.A., Gobernado M., Handke R., Laverdière M., Lee-Yang W., Merz W.G., Ostrosky-Zeichner L., Pemán J., Perea S., Perfect J.R., Pfaller M.A., Proia L., Rex J.H., Rinaldi M.G., Rodriguez-Tudela J.L., Schell W.A., Shields C., Sutton D.A., Verweij P.E. \& Warnock D.W. (2004) Journal of Clinical Microbiology, 42, 3475-3482.

[86]Fothergill A.W. (2012) Antifungal Susceptibility Testing, Clinical Laboratory and Standards Institute (CLSI) Methods, Interactions of Yeasts, Moulds, and Antifungal Agents: How to Detect Resistance, Springer Science+Business Media, LLC 2012.

[87]Slesiona S., Gressler M., Mihlan M., Zaehle C., Schaller M., Barz D., Hube B., Jacobsen I.D. \& Brock M. (2012) PLoS ONE 7(2), e31223.

[88]Beauvais A., Bozza S., Kniemeyer O., Formosa C., Balloy V., Henry C., Roberson R.W., Dague E., Chignard M., Brakhage A.A., Romani L. \& Latgé J.P. (2013) PLoS Pathogen 9(11), e1003716.

[89]Fernández-Torres B., Inza I. \& Guarro J. (2003) Antimicrobial Agents and Chemotherapy, 47(10), 3371-3372.

[90]Santos D.A., Barros M.E.S. \& Hamdan J.S. (2006) Journal of Clinical Microbiology, 44(1), 98-101.

[91]Deak E., Wilson S.D., White E., Carr J.H. \& Balajee S.A. (2009) PLoS One, 4, e7673.

[92]Deak E., Nelson M., Hernández-Rodríguez Y., Gade L., Baddley J., Momany M., Steele C. \& Balajee S.A. (2011) Virulence, 2(3), 200-207.

[93]Varanasi N.L., Baskaran I., Alangaden G.J., Chandrasekar P.H., Manavathu E.K. (2004) International Journal of Antimicrobial Agents, 23(1), 72-79.

[94]Fuller J., Schofield A., Jiwa S., Sand C., Jansen B. \& Rennie R. (2010) Journal of Clinical Microbiology, 48(2), 479-482.

[95]Arenderup M., Perkhofer S., Howard S.J., Garcia-Effron G., Vishukumar A., Perlin D. \& Lass-Flörl C. (2008) Antimicrobial Agents and Chemotherapy, 52, 3504-3511.

[96]Meletiadis J., Mouton J.W., Meis J.F.G.M., Bouman B.A., Donnelly P.J., Verweij P.E. \& Eurofung Network (2001) Journal of Clinical Microbiology, 39, 4256-4263.

[97]Kirk P.M. \& Cooper J. (2010) Index fungorum, CABI, Bio Science data base.

[98]Crous P.W., Gams W., Stalpers J.A., Robert V. \& Stegehuis G. (2004) Studies in Mycology, 50, 19-22.

[99]Vogelman B., Craig W.A. (1986) Journal of Pediatrics, 108, 835-840.

[100]Kahlmeter G., Brown D.F.J., Goldstein F.W., MacGowen A.P, Mouton J.W., Osterlund A., Rodloff A., Steinbakk M., Urbaskova P. \& Vatopoulos A. (2003) Journal of Antimicrobial Chemotherapy, 52, 145-148.

[101]Turnidge J. \& Paterson D.L. (2007) Clinical and Microbiology Reviews, 207, 391-408.

[102]Turnidge J., Kahlmeter G. \& Kronvall G. (2006) Clinical Micro- 
biology and Infection, 12, 418-425.

[103]Arendrup M.C., Kahlmeter G., Rodriguez-Tudela J.L., Donnelly J.P. (2009) Antimicrobial Agents and Chemotherapy, 53, 1628-1629.

[104]Arikan-Akdagli S. (2012) Annals of the New York Academy of Science, 1272(2912), 9-14.

[105]Pfaller M.A., Espinel-Ingroff A., Boyken L., Hollis R.J., Kroeger J., Messer S.A. \& Diekema D.J. (2011) Journal of Clinical Microbiology, 49(3), 845-850.

[106]Pfaller M.A., Diekema D.J., Ghannoum M.A., Rex J.H., Alexander B.D., Andes D., Brown S.D., Chaturvedi V., EspinelIngroff A., Fowler C.L., Johnson E.M., Knapp C.C., Motyl M.R., Ostrosky-Zeichner L., Sheehan D.J. \& Walsh T.J. (2009) Journal of Clinical Microbiology, 47, 3142-3146.

[107]Pfaller M.A. \& Diekema D.J. (2012) Journal of Clinical Microbiology, 50(9), 2846-2856.

[108]Arendrup M.C., Garcia-Effron G., Lass-Flörl C., Lopez A.G., Rodriguez-Tudela J.-L., Cuenca-Estrella M. \& Perlin D.S. (2010) Antimicrobial Agents and Chemotherapy, 54, 426-439.

[109]Meletiadis J., Mavridou E., Melchers W.J.G, Mouton J.W. \& Verweij P.E. (2011) Antimicrobial Agents and Chemotherapy, 56(5), 2524-2529.

[110]Cuenca-Estrella M. (2014) Clinical Microbiology and Infection. DOI: 10.1111/1469-0691.12495.

[111]Simjee S., Silley P., Werling H.O. \& Bywater R. (2008) Journal of Antimicrobial Chemotherapy, 61 (1), 228-229.

[112]Bywater R., Silley P., Simjee S. (2006) Veterinary Microbiology, 118, 158-159.

[113]Baddley J.W., Marr K.A., Andes D.R., Walsh T.J., Kauffman C.A., Kontoyiannis D.P., Ito J.I., Balajee S.A., Pappas P.G. \& Moser S.A. (2009) Journal of Clinical Microbiology, 47, 32713275.

[114]Garcia-Effron G., Dilder A., Alcazar-Fuoli L., Park S., Mellado E. \& Perlin D.S. (2008) Journal of Clinical Microbiology, 46, 1200-1206.

[115]Gulshan K. \& Moye-Rowley W. (2007) Eukaryotic Cell, 6, 1933-1942.

[116]Pfaller M.A., Boyken L., Hollis R.J., Kroeger J., Messer S.A,. Tendolkar S. \& Diekema D.J. (2009) Journal of Clinical Microbiology, 47, 3323-3325.

[117]Hodiamont C., Dolman K., Ten Berge I., Melchers W., Verweij P.E. \& Pajkrt D. (2009) Medical Mycology, 47, 217-220.

[118]Rodriguez-Tudela J.L., Cazar-Fuoli L., Mellado E., AstrueyIzquierdo A., Monzon A. \& Cuenca-Estrella M. (2008) Antimicrobial Agents and Chemotherapy, 52, 2468-2472.

[119]Snelders E., van der Lee H.A.L., Kuijpers J., Rijs A.J.M.M., Varga J., Samson R.A., Mellado E., Donders A.R., Melchers W.J. \& Verweij P.E. (2008) PLoS Medicine, 5(11), e219.

[120]Rocha E.M., Garcia-Effron G., Park S., Perlin D.S. (2007) Antimicrobial Agents Chemotherapy, 51, 4174-4176.

[121]MacCallum D.M., Whyte J.A. \& Odds F.C. (2005) Antimicrobial Agents and Chemotherapy, 49, 3697-3701.

[122]Hill J.A., Ammar R., Torti D., Nislow C. \& Cowen L.E. (2013) PLoS Genetics, 9(4), e1003390.

[123]Cacciapuoti A., Halpern J., Mendrick C., Norris C., Patel R. \&
Loebenberg D. (2006) Antimicrobial Agents and Chemotherapy, 50, 2587-2590.

[124]Dannaoui E., Lortholary O. \& Dromer F. (2004) Antimicrobial Agents and Chemotherapy, 48, 970-978.

[125]Bueid A., Howard S.J., Moore C.B., Richardson M.D., Harrison E., Bower B. \& Denning W. (2010) Journal of Antimicrobial Chemotherapy, 65, 2116-2118.

[126]Rodríguez-Tudela J.L., Arendrup M.C., Cuenca-Estrella M., Donnelly J.P. \& Lass-Flörl C. (2010) Drug News \& Perspectives, 23(2), 93-97.

[127]Stensvold C.R., Jørgensen L.N. \& Arendrup M.C. (2012) Current Fungal Infection Reports, 6, 178-191.

[128]Seyedmousavi S., Hashemi S.J., Zibafar E., Zoll J., Hedayati M.T., Mouton J.W., Melchers W.J.G. \& Verweij P.E. (2013) Azole-resistant Aspergillus fumigatus, Iran [letter]. Emerging Infectious Diseases, 19(5), 832-834.

[129]Morio F., Aubin G.G., Danner-Boucher I., Haloun A., Sacchetto E., Garcia-Hermoso D., Bretagne S., Miegeville M. \& Le Pape P. (2012) Journal of Antimicrobial Chemotherapy, 67, 1870-1873.

[130]Mellado E., De La Camara R., Buendia B., Rodriguez-Tudela J.L. \& Cuenca-Estrella M. (2013) Revista Iberoamerica de Micologia, 30, 64-68.

[131]Lockhart S.R., Frade J.P., Etienne K.A., Pfaller M.A., Diekema D.J. \& Balajee S.A. (2011) Antimicrobial Agents and Chemotherapy, 55, 4465-4468.

[132]Camps S.M., Rijs A.J., Klaassen C.H., Meis J.F., O'Gorman C.M. \& Dyer P.S. (2012) Journal of Clinical Microbiology, 50, 2674-2680.

[133]Enoch D.A., Idris S.F., Aliyu U., Micalleff C., Sule O. \& Karas J.A. (2014) Journal of Infection, 68(6), 507-526.

[134]Walker L.A., Gow N.A.R. \& Munro C.A. (2010) Fungal Genetics and Biology, 2, 117-126.

[135]Schoustra S. \& Punzalan D. (2012) Fungal Biology, 116(5), 630-636.

[136]Gifford D.R. \& Schoustra S. (2013) Journal of Theoretical Biology, 320, 124-130.

[137]He X., Li S. \& Kaminsky S.G.W. (2014) Eucariotic Cell, 13(2), 288-294.

[138]Schoustra S.E., Bataillon T., Gifford D.R. \& Kassen R. (2009) PLoS Biology 7(11), e1000250.

[139]Anderson J.B., Sirjusingh C., Sysed N. \& Lafayette S. (2009) Antimicrobial Agents and Chemotherapy, 53(5), 1931-1936.

[140]Mavridou E., Brüggemann R.J.M., Melchers W.J.G., Mouton J. \& Verweij P.E. (2010) Antimicrobial Agents and Chemotherapy, 54, 860-865.

[141]Mellado E., Garcia-Effron G., Alcazar-Fuoli L., CuencaEstrella M. \& Rodriguez-Tudela J.L. (2004) Antimicrobial Agents and Chemotherapy, 48, 2747-2750.

[142]Garcia-Effron G., Mellado E., Gomez-Lopez A., Alcazar-Fuoli L., Cuenca-Estrella M. \& Rodriguez-Tudela J.L. (2005) Antimicrobial Agents and Chemotherapy, 49, 2119-2121.

[143]Lionakis M.S., Lewis R.E., Torres H.A., Albert N.D., Raad I.I. \& Kontoyiannis D.P. (2005) Diagnostic Microbiology and Infectious Disease, 52(1), 15-20. 
[144]Escribano P., Recio S., Peláez T., González-Rivera M., Bouza E. \& Guinea J. (2011) Antimicrobial Agents and Chemotherapy, 56, 174-178.

[145]Mayr A. \& Lass-Flörl C. (2011) European Journal of Microbiology Research, 16, 153-157.

[146]Rambach G., Oberhauser H., Speth C. \& Lass-Flörl C. (2011) Medical Mycology, 49(8), 856-863.

[147]Pfaller M., Boyken L., Hollis R., Kroeger J., Messer S., Tendolkar S. \& Diekema D. (2011) Journal of Clinical Microbiology, 49, 586-590.

[148]Alanio A., Cabaret O., Sitterlé E., Costa J.-M-, Brisse S., Cordonnier C. \& Bretagne S. (2012) Antimicrobial Agents and Chemotherapy, 56, 4948-4950.

[149]Snelders E., Camps S.M.T., Karawajczyk A., Schaftenaar G., Kema G.H.J., van der Lee H.A., Klaassen C.H., Melchers W.J.G. \& Verweij P.E. (2012) PLoS ONE, 7, e31801.

[150]Czaika V., Nenoff P., Glöckner A., Fegeler W., Becker K. \& Schmalreck A.F. (2013) International Journal of Microbiology, 703905 . 